ARTICLE

\title{
Aneuploidy generates proteotoxic stress and DNA damage concurrently with p53-mediated post-mitotic apoptosis in SAC-impaired cells
}

Akihiro Ohashi ${ }^{1}$, Momoko Ohori ${ }^{1}$, Kenichi Iwai ${ }^{1}$, Yusuke Nakayama², Tadahiro Nambu${ }^{1}$, Daisuke Morishita ${ }^{1}$, Tomohiro Kawamoto², Maki Miyamoto³, Takaharu Hirayama1, Masanori Okaniwa', Hiroshi Banno1, Tomoyasu Ishikawa', Hitoshi Kandori ${ }^{4} \&$ Kentaro Iwata ${ }^{3}$

The molecular mechanism responsible that determines cell fate after mitotic slippage is unclear. Here we investigate the post-mitotic effects of different mitotic aberrationsmisaligned chromosomes produced by CENP-E inhibition and monopolar spindles resulting from Eg5 inhibition. Eg5 inhibition in cells with an impaired spindle assembly checkpoint (SAC) induces polyploidy through cytokinesis failure without a strong anti-proliferative effect. In contrast, CENP-E inhibition causes p53-mediated post-mitotic apoptosis triggered by chromosome missegregation. Pharmacological studies reveal that aneuploidy caused by the CENP-E inhibitor, Compound-A, in SAC-attenuated cells causes substantial proteotoxic stress and DNA damage. Polyploidy caused by the Eg5 inhibitor does not produce this effect. Furthermore, p53-mediated post-mitotic apoptosis is accompanied by aneuploidy-associated DNA damage response and unfolded protein response activation. Because Compound-A causes p53 accumulation and antitumour activity in an SAC-impaired xenograft model, CENP-E inhibitors could be potential anticancer drugs effective against SAC-impaired tumours.

\footnotetext{
${ }^{1}$ Oncology Drug Discovery Unit, Takeda Pharmaceutical Company Limited, 26-1, Muraoka-Higashi 2-chome, Fujisawa 251-8555, Japan. ${ }^{2}$ Biomolecular Research Laboratories, Takeda Pharmaceutical Company Limited, 26-1, Muraoka-Higashi 2-chome, Fujisawa 251-8555, Japan. ${ }^{3}$ DMPK Research Laboratories, Takeda Pharmaceutical Company Limited, 26-1, Muraoka-Higashi 2-chome, Fujisawa 251-8555, Japan. ${ }^{4}$ Drug Safety Research Laboratories, Pharmaceutical Research Division, Takeda Pharmaceutical Company Limited, 26-1, Muraoka-Higashi 2-chome, Fujisawa 251-8555, Japan. Correspondence and requests for materials should be addressed to A.O. (email: akihiro.ohashi@takeda.com).
} 
A ccurate control of chromosome segregation during mitosis is crucial for genomic stability. Chromosome segregation during mitosis involves dynamic interactions between spindle microtubules and kinetochores. These interactions are required for bipolar attachment between kinetochores and microtubules and subsequent alignment of sister chromatids to the metaphase plate. To maintain fidelity during chromosome segregation, the spindle assembly checkpoint (SAC) mechanism regulates the proper attachment of microtubules to kinetochores and the tension between the kinetochores of sister chromatids ${ }^{1}$. SAC prevents premature sister chromatid separation until the kinetochores of each duplicated chromosome pair have achieved bipolar attachment to the mitotic spindle ${ }^{2}$. Components of SAC, such as Bub1, Bub3, BubR1, Mad1 Mad2 and Mps1, preferentially localize at the kinetochores of unaligned chromosomes, where they produce a diffusible 'wait anaphase' signal ${ }^{1,3,4}$. This signal prevents the activation of the anaphase-promoting complex/ cyclosome, degradation of target proteins and progression from metaphase to anaphase. Disruption of the kinetochore assembly, attachment of spindle microtubules or SAC activity often leads to chromosome missegregation or premature mitotic exit, a process known as mitotic slippage ${ }^{5}$, and consequently generates aneuploidy, a hallmark of many solid tumours ${ }^{1,6-9}$.

Antimitotic therapeutics such as taxanes or vinca alkaloids, which suppress microtubule dynamics in the mitotic spindle to activate SAC, are widely used in the clinical treatment of cancer ${ }^{10}$. Although the detailed functional mechanisms of these drugs remain unclear, prolonged mitotic arrest appears to be one of the central mechanisms underlying the anti-proliferative activity of these drugs. Sustained mitotic arrest can provide more opportunities for antimitotic drugs to induce apoptosis ${ }^{11}$. Thus, to rescue cancer cells from mitotic death, mitotic slippage by SAC downregulation could bypass prolonged mitotic arrest before activating the apoptotic pathway in lesions refractory to antimitotic inhibitors ${ }^{5,12-16}$. To overcome the difficulties in the treatment of tumours resistant to current antimitotic drugs, next-generation mitotic inhibitors are expected to be effective against SAC-impaired and SAC-intact tumours.

CENP-E and Eg5 are mitotic spindle motor proteins of the kinesin superfamily ${ }^{17}$. Eg5 regulates centrosome separation and bipolar mitotic spindle formation ${ }^{18-20}$. CENP-E is localized at the kinetochores of chromosomes ${ }^{17,21}$ and controls chromosome alignment during metaphase by capturing the microtubule

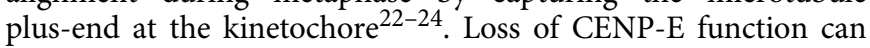
result in misaligned chromosomes during metaphase, leading to SAC activation ${ }^{23-30}$. Furthermore, CENP-E acts as a signaltransducing linker for BubR1-dependent SAC signalling by capturing it at spindle microtubule kinetochores ${ }^{29}$, indicating that CENP-E regulates mitotic progression and checkpoint activity. Recently, small-molecule inhibitors targeting mitotic components such as CENP-E and Eg5 have been developed as cancer therapeutics ${ }^{10,25,31-33}$. In preclinical studies, these mitotic inhibitors suppressed the proliferation and increased the apoptosis of cancer cells via various mitotic aberrations, monopolar mitotic spindles, chromosome misalignment, lagging chromosomes, centrosome fragmentation and cytokinesis failure. However, the molecular relationships between mitotic aberrations and suppression of proliferation remain unclear.

In this study, we investigated the molecular mechanisms by which CENP-E and Eg5 inhibition suppress cancer cell proliferation. To analyse these processes, we used a chemical inhibitor of CENP-E, Compound-A (Cmpd-A), as well as short interfering RNA (siRNA)-based approaches. We reveal that under SAC-defective conditions, aneuploidy caused by CENP-E inhibition triggers p53 activation after mitotic slippage, resulting in a post-mitotic decrease in proliferation. Polyploidy caused by
Eg5 inhibition does not produce this effect. Furthermore, we illustrate that aneuploidy-associated DNA damage response (DDR) and proteotoxic stress are accompanied by this postmitotic p53 activation. These findings will help to elucidate the molecular mechanisms linking chromosome instability and antiproliferation and promote translational research on mitotic inhibitors for cancer therapeutics.

\section{Results}

siCENP-E inhibits proliferation in a SAC-impaired condition. To evaluate the potential of CENP-E as a cancer therapeutic target, we investigated the molecular mechanisms by which CENP-E regulates cancer cell proliferation using siRNA-based approaches. Eg5 was used as a control because the loss of Eg5 function causes monopolar spindles and subsequent apoptosis during prolonged mitotic arrest ${ }^{18,19,34}$ (Supplementary Fig. 1a-d). siRNAs of both CENP-E (siCENP-E) and Eg5 (siEg5) induced BubR1 phosphorylation, which activates SAC (Fig. 1a). BubR1 knockdown (siBubR1) released both siCENP-Eand siEg5-transfected cells from prolonged mitotic arrest (Supplementary Fig. 1d), demonstrating that BubR1 monitored aberrant chromosome dynamics caused by siCENP-E or siEg5 and triggered SAC activation. We next determined whether SAC attenuation by siBubR1 could rescue siCENP-E- or siEg5transfected cells from apoptosis and recover their viability. siCENP-E or siEg5 transfection had a potent anti-proliferative effect and caused death in HeLa cells (Fig. 1b (open triangle) and (open circle) and Supplementary Fig. 1a,b,e) within $72 \mathrm{~h}$ after transfection. After double knockdown of Eg5 and BubR1 $($ siEg5 + siBubR1), cell viability was significantly restored with release from prolonged mitotic arrest (Fig. 1b (closed triangle) and Supplementary Figs 1d,e, 2 and 3). Conversely, the double knockdown of CENP-E and BubR1 (siCENP-E + siBubR1) did not restore cell viability (Fig. $1 \mathrm{~b}$ and Supplementary Figs 2 and 4). In siCENP-E + siBubR1 transfection experiments, the cells exited prolonged mitotic arrest (Supplementary Fig. 1d), and cell viability was rescued on day 2 (Fig. 1b). This result showed that the post-mitotic effects elicited anti-proliferation in these cells $24 \mathrm{~h}$ or later after mitotic death was bypassed. Crystal violet staining also confirmed that siBubR1 restored viability in siEg5transfected but not in siCENP-E-transfected cells (Fig. 1c and Supplementary Fig. 5a,b). These data demonstrate that although SAC attenuation can release both siCENP-E- and siEg5transfected cells from cell death during prolonged mitotic arrest, the post-mitotic effect suppresses proliferation in siCENP-E + siBubR1-transfected but not in siEg5+ siBubR1transfected cells.

Time-lapse microscopy revealed that siCENP-E + siBubR1 transfection allowed the cells to undergo cytokinesis, as did non-silencing siRNA (Fig. 1d). However, the presence of siEg5+ siBubR1 induced cytokinesis failure, generating polyploid cells. Polyploidy ( $>4 \mathrm{~N}$ DNA) increased in siEg5 + siBubR1-transfected cells but not in siCENP-E + siBubR1-transfected cells (Fig. 1e). Because Eg5 inhibition causes monopolar spindle formation, SAC attenuation by siBubR1 causes polyploidy in siEg5-transfected cells through cytokinesis failure. Conversely, in siCENP-E + siBubR1-transfected cells, lagging chromosomes or micronuclei, the hallmarks of chromosome missegregation, were observed frequently (Fig. 1f). To assess chromosome missegregation directly, fluorescence in situ hybridization was performed with Cy3-labelled probes for chromosome 17 (Chr-17) centromeres (Fig. 1g,h). In HeLa cells, three to four copies of Chr-17 were found in $97.1 \%$ (135/139 cells) of the control cells but only $55.4 \%$ (77/139 cells) of siCENP-E + siBubR1-transfected cells. Furthermore, the range of $\mathrm{Chr}-17$ copy numbers in 


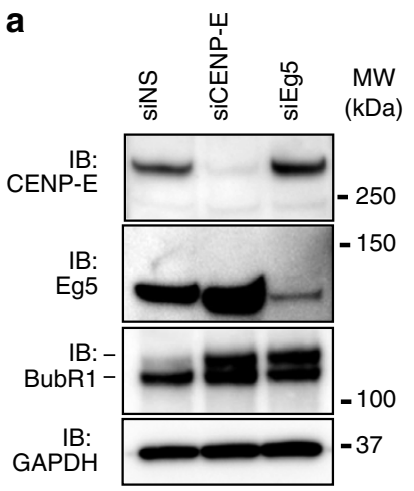

b

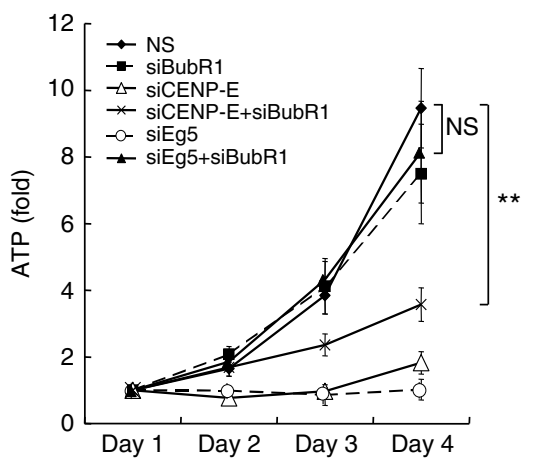

C

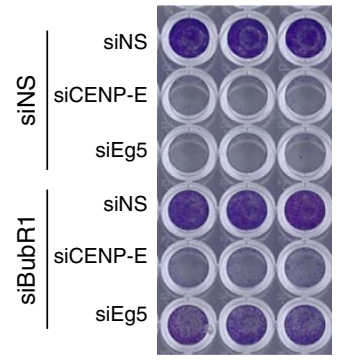

d
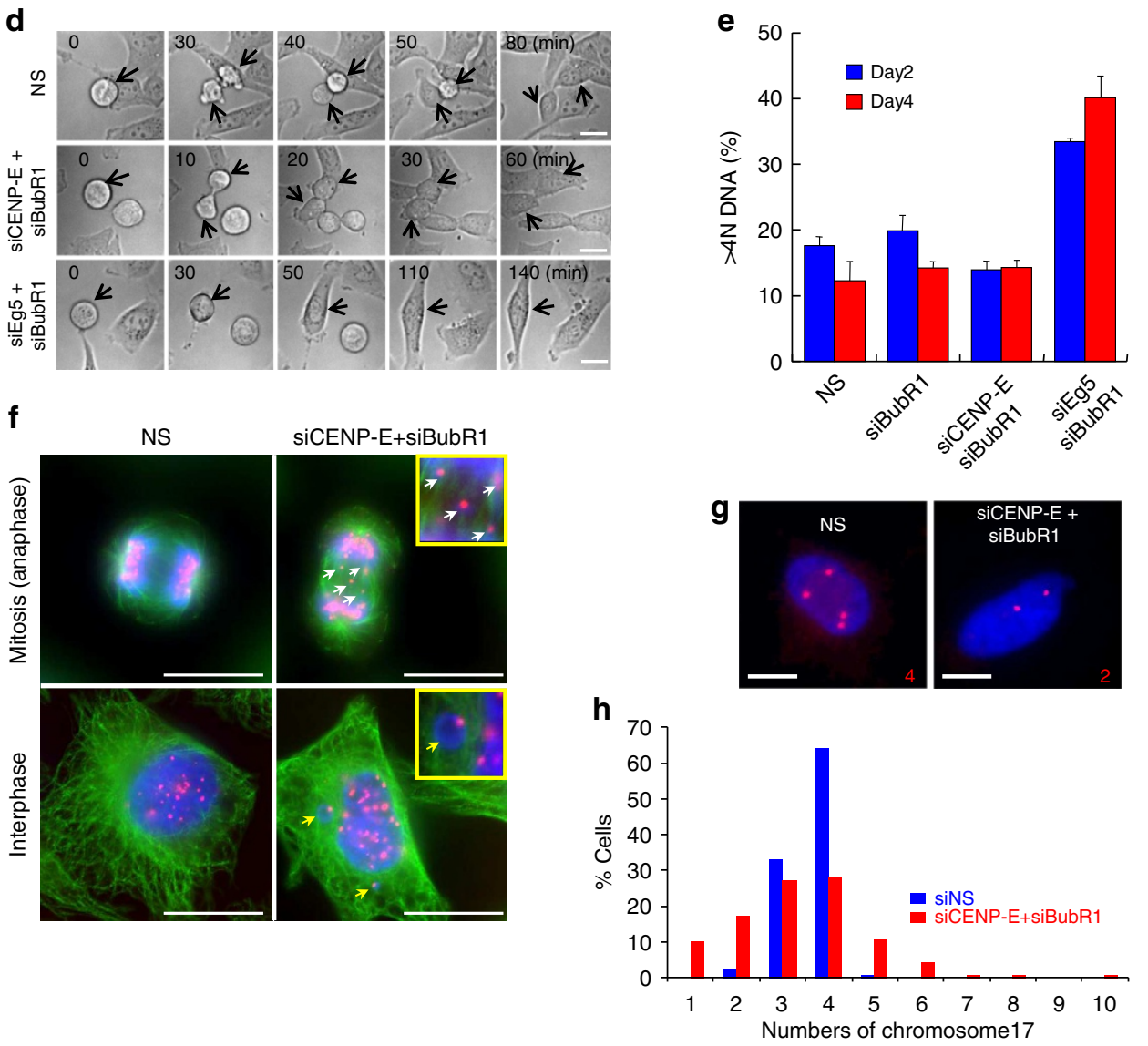

Figure 1 | Post-mitotic growth inhibition and chromosome missegregation caused by siCENP-E and siBubR1. (a) Immunoblotting of BubR1 in HeLa cells transfected with siCENP-E and siEg5. The upper band in the BubR1 rectangle represents phosphorylated BubR1. (b) Anti-proliferative effects of siCENP-E in siBubR1-transfected HeLa cells. Cells transfected with the indicated siRNA were collected on days 1-4 after transfection. Relative ATP levels were calculated based on luminescence in comparison with the day-1 luminescence value (control). The line plots represent mean \pm s.d. $(n=3)$. ATP levels on day 4 were statistically analysed using Student's $t$-test for comparison between siCENP-E + siBubR1- and siNS-transfected cells or between siEg5 + siBubR1- and siNS-transfected cells. Differences were considered significant at $P \leq 0.05\left(^{*}\right)$ and $P \leq 0.01$ ( $\left.^{\star \star}\right)$. (c) Representative images of crystal violet staining in HeLa cells transfected with the indicated siRNAs. Cells were collected on day 3 after transfection for crystal violet staining. (d) Time-lapse microscopy of HeLa cells transfected with siNS (upper row), siCENP-E + siBubR1 (middle row) or siEg5 + siBubR1 (lower row). Time point $t=0$ indicates the onset of mitosis. Arrows indicate cells undergoing mitosis. White bars indicate $20 \mu \mathrm{m}$. (e) Quantification of the $>4$ N DNA population was determined by FACS analysis on days 2 and 4 after siRNA transfection. Data are presented as mean \pm s.d. $(n=3)$. (f) Micronuclei and lagging chromosomes in siCENP-E- and siBubR1transfected HeLa cells. Green, red and blue signals indicate $\alpha$-tubulin, CENP-B and DAPI, respectively. White and yellow arrows denote the micronuclei and lagging chromosomes, respectively. White bars indicate $20 \mu \mathrm{m}$. (g,h) Fluorescence in situ hybridization (FISH) analysis using centromeric probes. FISH analysis using centromeric probes for Chr-17 was performed $48 \mathrm{~h}$ after siRNA transfection (red, Chr-17 centromeres; blue, DNA). Representative FISH images of cells transfected with siNS or siCENP-E + siBubR1 (g). White bars indicate $10 \mu \mathrm{m}$. The graph shows quantitative FISH analysis (blue, siNS-transfected cells $(n=139)$; red, siCENP-E + siBubR1-transfected cells $(n=139) ; \mathbf{h})$. 
siCENP-E + siBubR1-transfected cells was wider (1-10 chromosomes) than that in the control cells. To investigate the effect of asymmetric chromosome segregation on proliferation, we induced cytokinesis failure in siCENP-E + siBubR1-transfected cells. Cytokinesis failure produces polyploid daughter cells with twofold more chromosomes than their parental cells. Therefore, it does not result in aneuploid daughter cells with copy-number variations for each chromosome caused by asymmetric chromosome segregation. Mitotic kinesin-like protein 2 (MKLP2), also known as kinesin superfamily protein $20 \mathrm{~A}$ (KIF20A $)^{17}$, is involved in the localization of several mitotic proteins at the central spindle where they regulate cytokinesis ${ }^{35}$. Knockdown of MKLP2 (siMKPL2) induced cytomegalic phenotypes in siCENP-E + siBubR1-transfected cells. Among the cells with triple knockdown of CENP-E, BubR1 and MKLP2 (siCENP$\mathrm{E}+$ siBubR1 + siMKLP2), the population with $>4 \mathrm{~N}$ DNA cells was significantly larger than that among siCENP-E + siBubR1transfected cells (Fig. 2a,b and Supplementary Fig. 6a-c). In the polyploid cells induced by siCENP-E + siBubR1 + siMKPL2, similar to those induced by $\operatorname{siEg} 5+$ siBubR 1 , cell viability was restored to a significantly higher level than that in the aneuploid cells induced by siCENP-E + siBubR1 (Fig. 2c,d and Supplementary Fig. 6d,e). Supporting their proliferative recovery, caspase-3/7 activity was significantly lower in siCENP$\mathrm{E}+$ siBubR1 + siMKPL2-transfected cells than in siCENP$\mathrm{E}+$ siBubR1-transfected cells (Fig. 2e). We also determined the effect of other SAC-associated genes on cell viability in siCENPE-transfected cells compared with siEg5-transfected cells. Knockdown of Mad2 and Bub3 significantly restored the viability of siEg5-transfected cells. The viability of siCENP-Etransfected cells was unaffected by the knockdown of any SAC-associated gene (Fig. 2f,g and Supplementary Fig. 7a-f). In summary, CENP-E inhibition coupled with SAC attenuation increases asymmetric chromosome segregation, which is responsible for the post-mitotic anti-proliferative effect.

Aneuploidy upregulates the $\mathbf{p} 53$ pathway in SAC-impaired cells. To determine which signalling pathways contribute to the post-mitotic anti-proliferative effect of asymmetric chromosome segregation, comprehensive gene expression analysis was performed by microarray analysis (Fig. 3a and Supplementary Fig. 8a). Because the single knockdown of CENP-E or Eg5 caused substantial cell death within 3 days after transfection (Fig. $1 \mathrm{~b}$ and Supplementary Figs 1-4), cell lysates from these cells could not be used in the microarray, reverse transcription-PCR (RT-PCR) or immunoblotting experiments. Thus, siNS-transfected cells were used as a control. Compared with the control cells, 52 of the 343 p53-regulated transcripts were upregulated in siCENP-E + siBubR1-transfected cells, whereas 27 and 13 transcripts were upregulated in siEg5 + siBubR1- and siBubR1-transfected cells, respectively (Fig. 3b and Supplementary Fig. 8b,c). RT-PCR analysis also confirmed that GADD45A expression increased in siCENP-E + siBubR1-transfected cells (Fig. 3c). Although HeLa cells possess wild-type $p 53$, human papillomavirus (HPV)-encoded E6/E7 genes downregulate the expression of the p53 protein. However, p53 expression was increased in siCENP-E + siBubR1transfected HeLa cells, consistent with the microarray data (Fig. 3d). We also confirmed the upregulation of p53 by siCENP$\mathrm{E}+$ siBubR1 in the HPV-negative but p53-wild-type cancer cell lines HCT116 and H460 (Supplementary Fig. 8d-f). This result indicates that $\mathrm{p} 53$ accumulation induced by siCENP-E + siBubR1 is independent of the HPV-associated p53 degradation machinery. Thus, in our model, HPV-encoded E6/E7 had little effect on the detected p53 accumulation. We mainly used HeLa cells in the subsequent experiments because of their technical availability for various assays. Pathway analysis using 434 upregulated genes, 317 downregulated genes and p53 itself also confirmed the central role of the p53 network in cell death and survival (Fig. 3e). Because p53 and caspase-3/7 expression was elevated concurrently (Fig. 2c,e and Supplementary Fig. 8g), we induced additional p53 knockdown in siCENP-E + siBubR1transfected cells to examine the relationship between p53 and caspase-3/7 elevation. Compared with the control cells, a twofold increase in caspase-3/7 activity was detected in siCENP-E + siBubR1-transfected cells. Caspase-3/7 activity in siCENP-E + siBubR1 + sip53-transfected cells was 1.3-fold lower (Fig. $3 \mathrm{f}$ and Supplementary Fig. 8h). Conversely, in the p53-defective cell line SK-BR3 and p53-knockout HCT116 cells, siCENP-E + siBubR1 did not induce caspase-3/7 activation (Fig. 3g,h and Supplementary Fig. 8i). CENP-E knockdown alone activated caspase-3/7 in SK-BR3 and HeLa cells (Figs 2e and 3g), demonstrating that caspase-3/7 activation during mitotic death in cells with intact SAC is regulated in a p53-independent manner. These data suggest that chromosome missegregation by CENP-E inhibition coupled with SAC attenuation activates p53 pathways, causing a post-mitotic anti-proliferative effect following mitotic slippage.

Aneuploidy generates replication stress-mediated DSBs. A novel small-molecule inhibitor of CENP-E, Cmpd-A ( + - $-\mathrm{N}-[7-$ cyano-1,1-dioxido-6-(trifluoromethyl)-2,3-dihydro-1-benzothiophen-3-yl]-N-[2-(dimethylamino)ethyl]-3-(4-fluoro-3-methylphenyl)-5-methoxyimidazo[1,2-a]pyridine-2-carboxamide), was developed (Fig. 4a, Supplementary Methods). The seed compounds were discovered by high-throughput chemical library screening with ATPase assays. Cmpd-A represents a chemically optimized molecule with the seed compounds. Cmpd-A inhibits the ATPase activity of the CENP-E-motor domain, with an $\mathrm{IC}_{50}$ value of $2.2 \mathrm{nM}$. It does not inhibit Eg5 or KHC kinesins or any of the other 36 tested kinases (Supplementary Figs 9 and 10). Treatment of HeLa cells with Cmpd-A caused chromosome misalignment, $\mathrm{pHH} 3$ accumulation, potent growth inhibition and cell death within $48 \mathrm{~h}$ (Fig. $4 \mathrm{a}-\mathrm{d}$ and Supplementary Fig. 11a, $\mathrm{GI}_{50}=80 \mathrm{nM}$ ). Given that CENP-E also functions as a signaltransducing linker in SAC signalling ${ }^{29}$, inhibition of this enzyme by Cmpd-A may induce more prolonged mitotic arrest than siCENP-E alone (Supplementary Fig. 11b,c). We analysed the effect of the pharmacological inhibition of CENP-E with Cmpd-A in comparison with the chemical inhibition of Eg5 by ispinesib. Supporting the results of our siRNA studies, treatment with Cmpd-A, but not with ispinesib, had a potent anti-proliferative effect in siBubR1-transfected HeLa cells (Fig. 4c,d and Supplementary Fig. 11d-f). Whereas Cmpd-A or ispinesib monotherapy caused substantial cell death within $48 \mathrm{~h}$ (Fig. 4b,c and Supplementary Fig. 11a), p53 accumulation was not detected in these cells. These results indicate that unlike aneuploidyassociated post-mitotic cell death, mitotic cell death caused by CENP-E or Eg5 inhibition is independent of p53. Samples taken $72 \mathrm{~h}$ after these single-agent treatments could not be used in further assays because of the high levels of cell death (Fig. $4 \mathrm{~b}-\mathrm{d}$ ). Thus, siNS + dimethylsulphoxide (DMSO)-treated cells were used as a control. In agreement with the results of our siRNA-based studies, substantial p53 accumulation was detected in siBubR1-transfected cells treated with Cmpd-A (siBubR1 + Cmpd-A; Fig. 4e). This was not observed in siBubR1-transfected cells treated with ispinesib (siBubR1 + ispinesib). To assess the effects of p53 on the anti-proliferative activity of Cmpd-A, we conducted cell viability assays in p53 isogenic HCT116 cell lines. Cmpd-A treatment without siBubR1 had similar anti-proliferative effects in p53-wild-type 
a

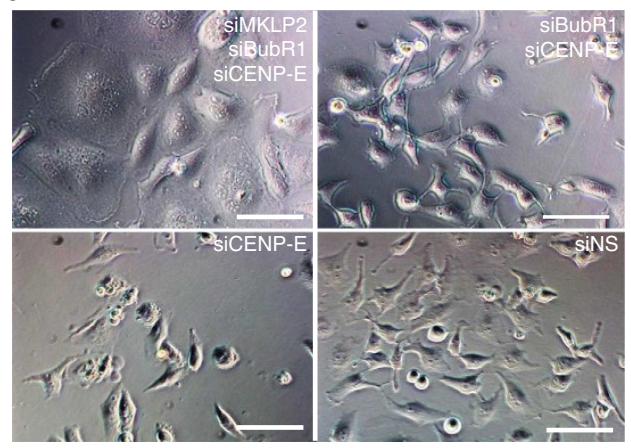

b

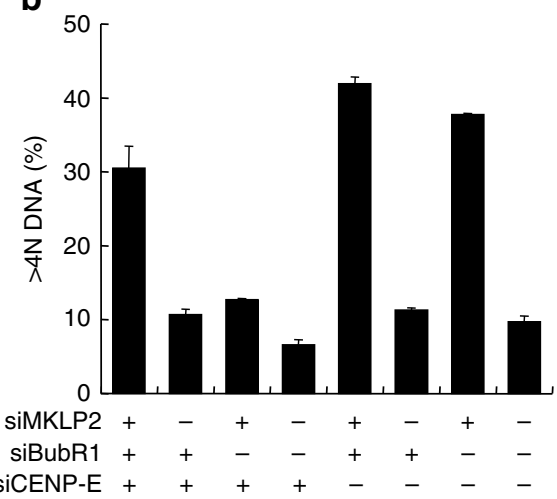

C

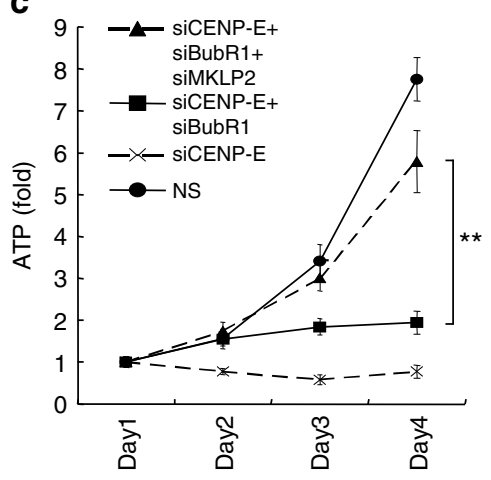

d

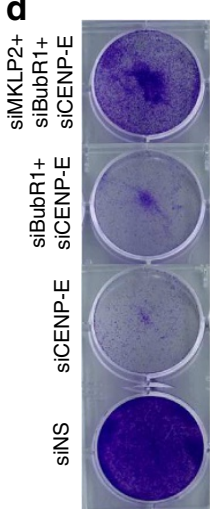

e

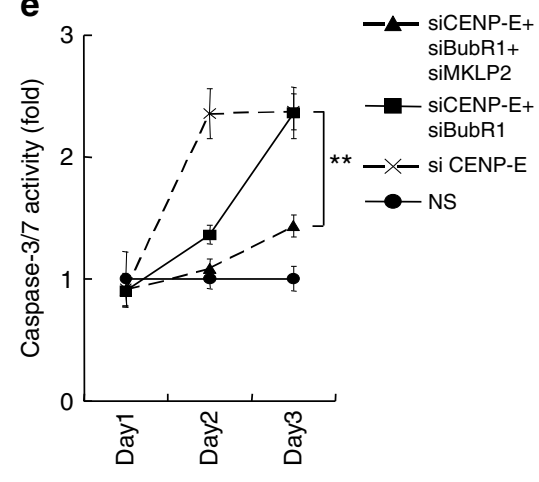

f

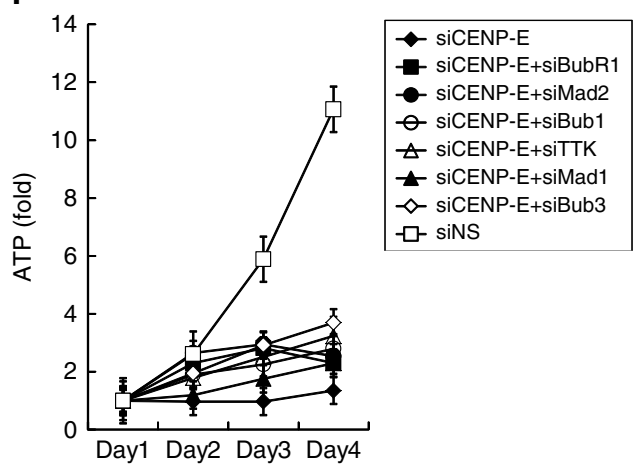

g

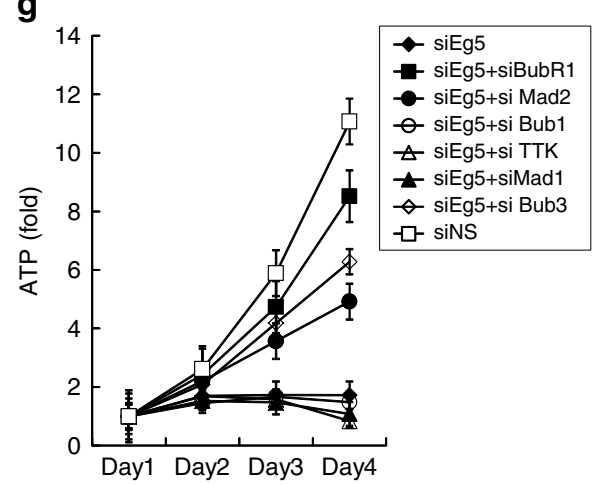

Figure 2 | Failure of cytokinesis restores cell viability in siCENP-E + siBubR1-transfected cells. (a) Phase-contrast microscopy images of CENP-E, BubR1 and MKLP2 triple-knockdown cells. Images were acquired 3 days after siRNA transfection. White bars indicate $100 \mu \mathrm{m}$. (b) The $>4 \mathrm{~N}$ DNA population was quantified by FACS analysis $48 \mathrm{~h}$ after siRNA transfection. Data are presented as mean \pm s.d. $(n=3)$. (c) Proliferative effect of siMKLP2 in siCENP-

$\mathrm{E}+$ siBubR1-transfected HeLa cells. The cells were transfected with the indicated siRNAs. Cell growth was evaluated as indicated in Fig. 1b. The line plots represent mean \pm s.d. $(n=3)$. ATP levels on day 4 were statistically analysed using Student's $t$-test for comparisons between siCENP-E + siBubR1 + siMKLP2and siCENP-E + siBubR1-transfected cells. Differences were considered significant at $P \leq 0.05\left(^{*}\right)$ and $P \leq 0.01\left(^{\star \star}\right)$. (d) Representative images of crystal violet staining in HeLa cells transfected with the indicated siRNAs. Cells were collected on day 3 after transfection. (e) Apoptotic effect of siMKLP2 in siCENP$\mathrm{E}+$ siBubR1-transfected HeLa cells. Relative caspase-3/7 activities were calculated based on the luminescence in comparison with the day- 1 luminescence value (control). The line plots represent mean \pm s.d. $(n=3)$. Caspase-3/7 activities on day 3 were statistically analysed using Student's $t$-test for comparison between siCENP-E + siBubR1 + siMKLP2- and siCENP-E + siBubR1-transfected cells. Differences were considered significant at $P \leq 0.05\left({ }^{\star}\right)$ and $P \leq 0.01\left({ }^{\star \star}\right)$. $(\mathbf{f}, \mathbf{g})$ Proliferative effect of the knockdown of SAC-associated genes in siCENP-E- or siEg5-transfected HeLa cells. The cells were transfected with siCENP-E (e) or siEg5 (f) in combination with the indicated siRNAs. Cell growth was evaluated as indicated in Fig. 1b. The line plots represent mean \pm s.d. $(n=3$ ).

and p53-knockout HCT116 cells, whereas Cmpd-A treatment with siBubR1 was more effective in p53-wild-type cells than in p53-knockout cells (Fig. 4f). These results also support the assumption that p53 contributes to aneuploidy-associated antiproliferative effects after mitotic slippage.

Similar to siCENP-E + siBubR1-transfected cells (Supplementary Figs $8 \mathrm{e}$ and 12a), we detected p53 phosphorylation at Ser-15 in
siBubR1 + Cmpd-A-treated cells (Fig. 4e). The increase in $\gamma \mathrm{H} 2 \mathrm{AX}$ (a marker of DNA double-strand breaks (DSBs)) levels also paralleled p53 accumulation in these cells (Supplementary Fig. 12b,c), suggesting that DSBs were generated in these cells. More directly, the neutral comet assay revealed that DNA tails, which indicate DSBs, were significantly longer in siBubR1+ Cmpd-A-treated cells (Fig. 5a,b and Supplementary Fig. 12d). 
Furthermore, inhibitors of ATM (Ku55933) and ATR $(\mathrm{VE}-821)^{36,37}$ suppressed p53 phosphorylation and p21 expression (Supplementary Fig. 12e). To summarize, chromosome missegregation caused by CENP-E inhibition and SAC attenuation generated DSBs, and the activated DDR contributed to p53 phosphorylation by ATM and ATR. We next determined whether DSBs in aneuploid cells are generated during the $S$ phase. Fluorescence-activated cell sorting (FACS) analysis revealed that BrdU-positive cells (in the $\mathrm{S}$ phase) were significantly reduced by siBubR1 + Cmpd-A treatment, whereas neither G1 nor G2 arrest was observed (Fig. 5c,d and Supplementary Fig. 13a,b).
Conversely, BrdU incorporation in siBubR $1+$ ispinesib-treated polyploid cells did not significantly decrease compared with that in siBubR1 + DMSO-treated cells (Supplementary Fig. 13a,b). Immunoblotting also revealed that the levels of phosphorylated MCM2 at Ser40, MCM2 and Cyclin A, which are DNA replication regulatory proteins, were decreased in siBubR $1+$ Cmpd-A-treated cells (Fig. 5e), whereas the level of the $\mathrm{G} 2$ phase protein Cyclin B was not reduced. To determine the correlation between impairment of DNA replication and DSB accumulation, we performed immunofluorescence 5-ethynyl-2'-deoxyuridine (EdU) incorporation assays for 53BP1. As DSBs were generated mainly a

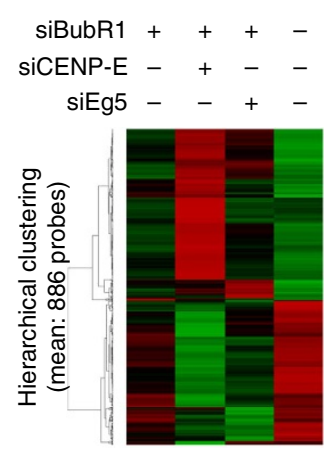

b

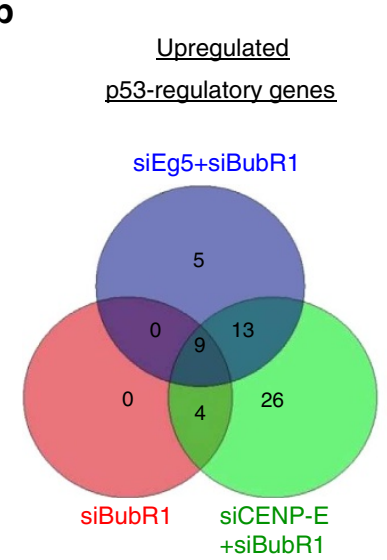

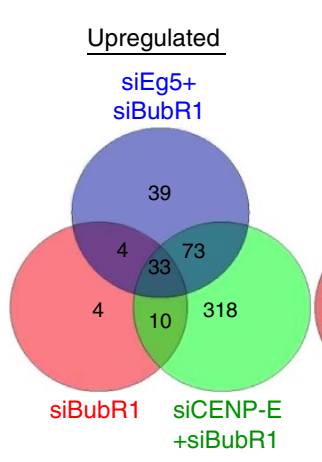
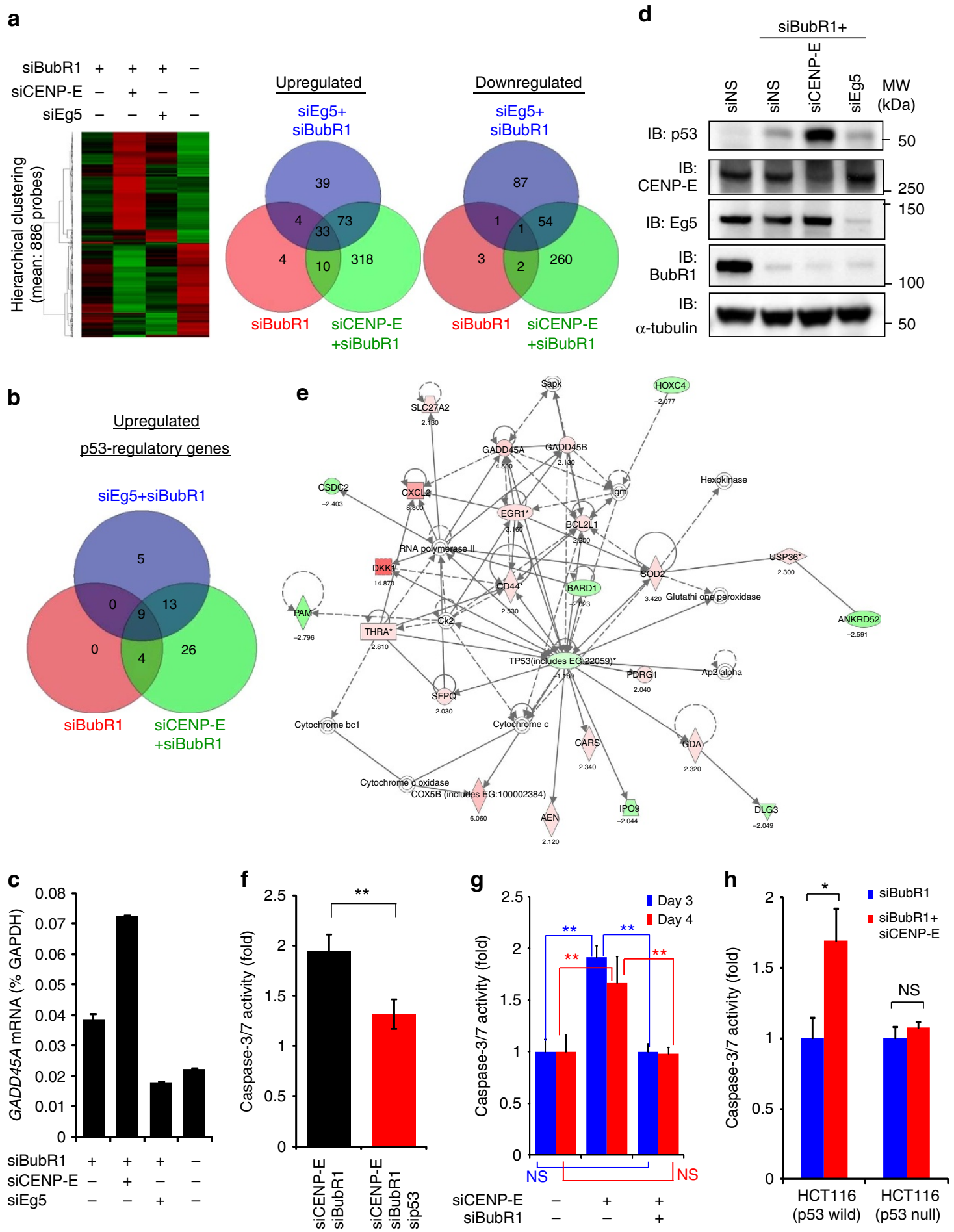
between 24 and $48 \mathrm{~h}$ after Cmpd-A treatment (Supplementary Fig. 12c), siBubR1-transfected HeLa cells were first treated with Cmpd-A for $24 \mathrm{~h}$. Then, EdU was added, and the cells were incubated for the next $24 \mathrm{~h}$ without Cmpd-A (Fig. 5f). Immunofluorescence analysis revealed 53BP1 foci in EdUnegative cells (Fig. 5f, red arrows) but not in EdU-positive cells (Fig. 5f, white arrows). As the caspase inhibitor ZVAD-FMK had little effect on 53BP1 foci (Supplementary Fig. 13c), DSBs detected in these cells were not the consequence of apoptosis-inducing DNA breaks. In summary, chromosome missegregation accelerated by siBubR1 + Cmpd-A treatment reduces replication activity during the $S$ phase; this replication stress appears to be responsible for the generation of aneuploidy-associated DSBs.

Aneuploidy induces proteotoxic stress and UPR activation. Recent studies have revealed that the excess of proteins produced by additional chromosomes disturbs the protein quality control systems in aneuploid yeast cells, leading to growth deficiency ${ }^{38,39}$. We investigated whether aneuploidy-associated proteotoxic stress can be observed in siBubR1 + Cmpd-A-treated cells. Electron microscopy analysis detected the expansion of the endoplasmic reticulum (ER; Fig. 6a, upper panels) and increased numbers of lysosomes (Fig. 6a, lower panels) in siBubR1 + Cmpd-A-treated HeLa cells relative to controls. Furthermore, accumulation of insoluble sequestosome 1 (p62), p62 aggresome formation ${ }^{40,41}$ and autophagic vesicle-associated form of light-chain 3B (LC3B-II) were also prominently detected in siBubR $1+$ Cmpd-A-treated cells but not in siBubR1 + ispinesib- or siBubR1 + DMSO-treated cells (Fig. $6 \mathrm{~b}$ and Supplementary Fig. 14a-c). Although the effects of Cmpd-A were relatively mild, siBubR $1+$ Cmpd-A treatment also caused p53 and LC3B induction in MCF10A untransformed mammary gland epithelial cells (Supplementary Fig. 15a-c). These results illustrate that aneuploidy-mediated protein homeostasis imbalance produces aggresomes of misfolded proteins. Then, autophagy is triggered to alleviate aneuploidy-associated proteotoxic stress by degrading abnormal or potentially toxic proteins ${ }^{42,43}$. We next established reporter assay systems for unfolded protein response (UPR) markers, namely the ER luminal chaperone BiP, ER membrane-associated protein ATF6 and UPR mediators XBP1 and $\mathrm{Chop}^{44}$. The reporter assays revealed increased levels of all tested markers in siBubR1 + Cmpd-Atreated cells (Fig. 6c, red bars) but not in siBubR1 + ispinesibtreated cells (Fig. 6c, green bars). An XBP1 splicing assay ${ }^{45}$ confirmed UPR activation in these cells (Fig. 6d and Supplementary Fig. 16a). Similar to the results obtained with
Cmpd-A or ispinesib, siCENP-E + siBubR1-transfected cells, but not siEg5 + siBubR1-transfected cells, also exhibited 53BP1 foci, LC3B-II formation, p62 accumulation, UPR reporter activation and XBP1 splicing (Supplementary Fig. 14b-e).

We also examined the effect of extraneous proteotoxic stress caused by the proteasome inhibitor MG132 on apoptosis and cell viability in siBubR $1+$ Cmpd-A- and siBubR $1+$ ispinesib-treated cells (Fig. 7a). Immunoblotting revealed that although MG132 treatment induced the accumulation of global ubiquitinated proteins in siBubR1 + DMSO- and siBubR1 + ispinesib-treated cells in a concentration-dependent manner, smaller amounts of ubiquitinated proteins were accumulated in siBubR1 + Cmpd-Atreated cells (Fig. 7b). L-Azidohomoalanine incorporation assays revealed that the levels of newly synthesized proteins were considerably lower in siBubR $1+$ Cmpd-A-treated cells than in siBubR1 + DMSO- or siBubR1 + ispinesib-treated cells (Fig. 7c). These data indicate that under substantial aneuploidy-associated proteotoxic stress in siBubR $1+$ Cmpd-A-treated cells, UPR appears to downregulate the protein translation machinery to relieve this stress. As a result, these cells accumulate fewer ubiquitinated proteins in response to MG132. Accordingly, MG132 treatment neither enhanced caspase-3/7 levels nor caused growth inhibition in these aneuploid cells (Fig. $7 \mathrm{~d}$, red bars, and Fig. 7e, red line). Conversely, the levels of newly synthesized proteins and MG132-induced ubiquitinated proteins in siBubR1 + ispinesib- and siBubR1 + DMSO-treated cells were similar, although they were higher than those in siBubR1+ Cmpd-A-treated cells (Fig. 7b,c). Moreover, MG132 treatment significantly elevated caspase-3/7 activity in siBubR1 + ispinesibtreated cells in a concentration-dependent manner (Fig. $7 \mathrm{~d}$, green bars) and reduced viability to the level of siBubR $1+$ Cmpd-Atreated cells (Fig. 7e, green and red lines). These results demonstrate that at least in these models, proteotoxic stress appears to be a key event; they also explain the different cell fates of aneuploid and polyploid cells after mitotic slippage. Although the detailed mechanisms remain unclear, polyploid cells may overdrive the protein quality control machineries to adapt to marked changes in protein homeostasis caused by the doubling of chromosome number after mitotic slippage. Such cells may be more sensitive to the modulation of protein homeostasis by proteasome inhibition.

Cmpd-A exhibits antitumour activity in SAC-attenuated cells. To examine spontaneous SAC attenuation, we assessed BubR1 expression in several cancer cell lines. Caki-1 cells (p53-wild-type

\footnotetext{
Figure 3 | Comprehensive gene expression analysis in siCENP-E + siBubR1-transfected cells. (a) Microarray analysis of the transcriptome of HeLa cells transfected with the indicated siRNAs. (Left) Hierarchical cluster analysis of 886 transcript expression profiles. Each row represents a single transcript. Red and green: relatively high and low expression, respectively. (Right) Venn diagrams. 'Upregulated' and 'Downregulated': $>2$-fold increase and $>2$-fold decrease, respectively, in the expression of the indicated transcripts compared with siNS-transfected cells. (b) Venn diagram-related to p53 pathways. 'Upregulated': > 2-fold increase in the levels of 343 p53-regulated transcripts compared with siNS-transfected cells. (c) Quantitative RT-PCR analysis of GAD45A in HeLa cells $72 \mathrm{~h}$ after transfection. GAD45A expression ratios were quantified using GAPDH expression. Data are presented as mean \pm s.d. $(n=3)$. (d) Protein expression of p53 in siCENP-E + siBubR1-transfected cells on day 3 after transfection. (e) Pathway analysis. The network was generated by Ingenuity Pathway Analysis (Ingenuity Systems, http://www.ingenuity.com) using p53 and 434 upregulated and 317 downregulated genes in siCENP$\mathrm{E}+$ siBubR1-transfected cells. (f) Effect of p53 on caspase-3/7 activity in siCENP-E + siBubR1-transfected cells. Black and red bars: siCENP-E + siBubR1transfected and siCENP-E + siBubR1 + sip53-transfected cells, respectively. Cells were collected on day 3 after transfection. Caspase-3/7 activities were evaluated as indicated in Fig. 2e. Data are presented as mean \pm s.d. $(n=3)$. Student's $t$-test was used to compare siCENP-E + siBubR1 + sip53- and siCENP$\mathrm{E}+$ siBubR1-transfected cells. Differences were considered significant at $P \leq 0.05\left(^{*}\right)$ and $P \leq 0.01\left(^{\star \star}\right)$. ( $\mathbf{g}$ ) Effect of p53 on caspase-3/7 activity in siCENP$E+$ siBubR1-transfected SK-BR3 cells. Cells were collected on days 3 (blue) and 4 (red) after transfection. Caspase-3/7 activities were evaluated as indicated in Fig. 2e. Data are presented as mean \pm s.d. $(n=3)$. Statistical analysis was performed using Student's $t$-test. Differences were considered significant at $P \leq 0.05\left(^{*}\right)$ and $P \leq 0.01\left(^{* *}\right)$. (h) Caspase-3/7 activation by siCENP-E + siBubR1 in p53-wild-type and p53-knockout HCT116 cells on day 3 after transfection. Caspase-3/7 activities were evaluated as indicated in Fig. 2e. Data are presented as mean \pm s.d. $(n=3)$. Statistical analysis was performed using Student's $t$-test. Differences were considered significant at $P \leq 0.05\left(^{*}\right)$ and $P \leq 0.01\left(^{* *}\right.$ ).
} 
a

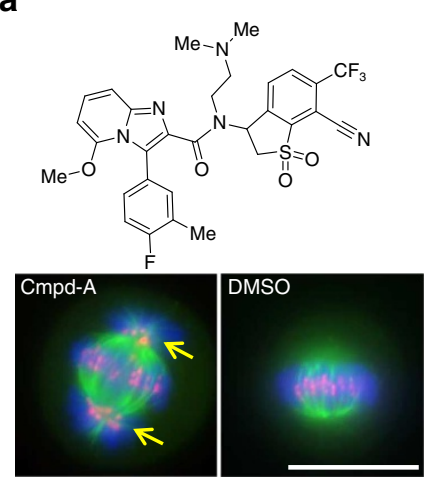

C

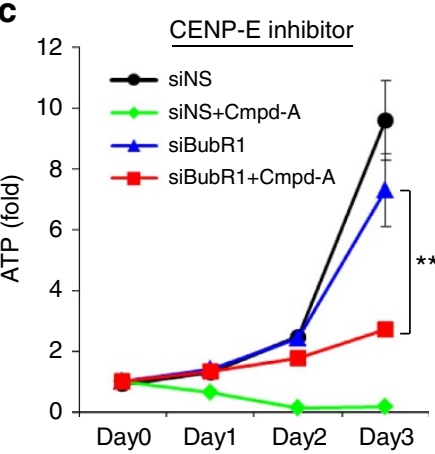

e

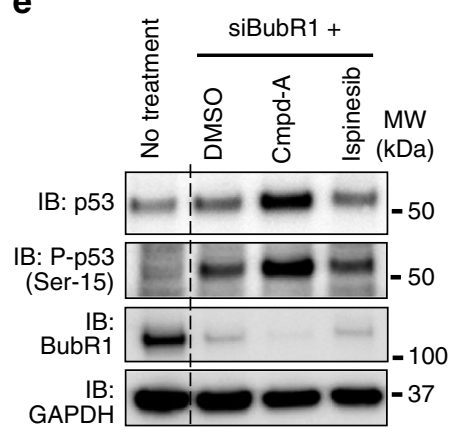

b
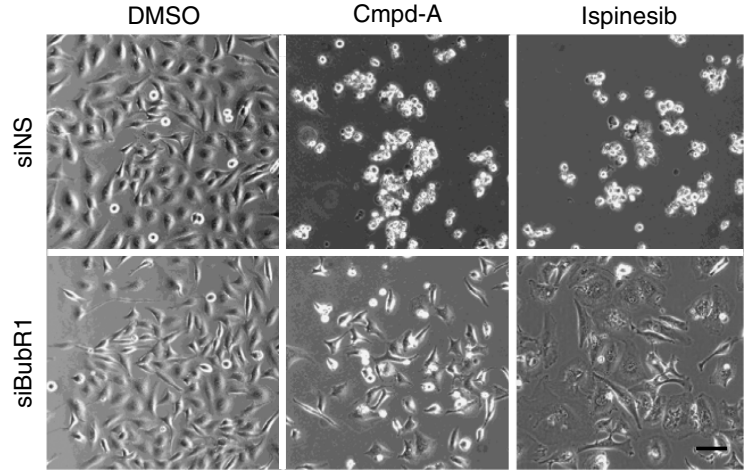

d
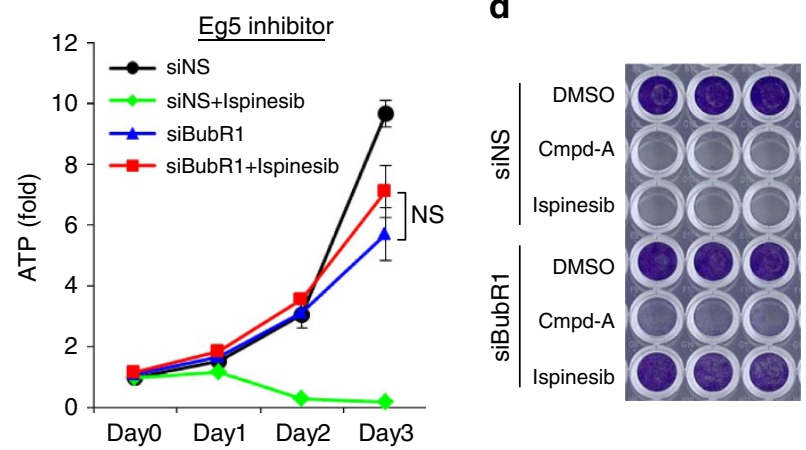

f

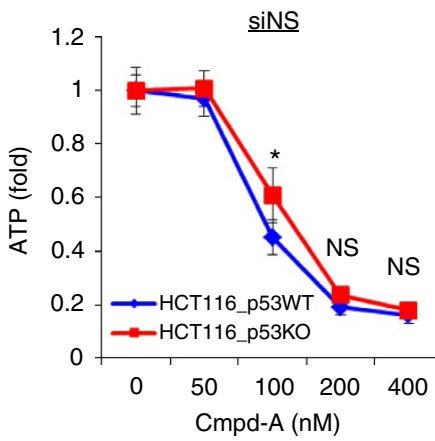

Figure 4 | Pharmacological inhibition of CENP-E causes p53-associated suppression of proliferation in SAC-impaired cells but not in SAC-intact cells. (a) Chemical structure of Cmpd-A (Upper) and misaligned chromosomes in HeLa cells with Cmpd-A treatment (Lower). Green, red and blue signals indicate $\alpha$-tubulin, CENP-B and DAPI, respectively. Yellow arrows denote the misaligned chromosomes. White bars indicate $20 \mu \mathrm{m}$. (b) Phase-contrast microscopy images of siNS- and siBubR1-transfected cells treated with DMSO, Cmpd-A and ispinesib. Twenty-four hours after siRNA treatment, the cells were treated with DMSO, Cmpd-A $(200 \mathrm{nM})$ or ispinesib $(10 \mathrm{nM})$. Images were acquired $48 \mathrm{~h}$ after drug treatment. The black bar indicates $100 \mu \mathrm{m}$. (c) Anti-proliferative effect of $\mathrm{Cmpd}-\mathrm{A}$ and ispinesib in siBubR1-transfected HeLa cells. Twenty-four hours after siRNA treatment, the cells were treated with DMSO, Cmpd-A (200 nM) or ispinesib $(10 \mathrm{nM})$ (day 0). Cell growth was evaluated as indicated in Fig. 1b. The line plots represent mean $\pm \mathrm{s} . \mathrm{d}$. $(n=3)$. ATP levels on day 3 were statistically analysed using Student's $t$-test for comparison between siBubR1 + Cmpd-A- and siBubR1-transfected cells and between siBubR1 + ispinesib- and siBubR1-transfected cells. Differences were considered significant at $P \leq 0.05\left(^{*}\right)$ and $P \leq 0.01\left(^{\star \star}\right)$. (d) Representative images of crystal violet staining in siNS- and siBubR1-transfected cells treated with DMSO, Cmpd-A and ispinesib. Twenty-four hours after siRNA treatment, the cells were treated with DMSO, Cmpd-A $(200 \mathrm{nM})$ or ispinesib $(10 \mathrm{nM})$. Cells were collected 5 days after drug treatment for crystal violet staining. (e) Immunoblotting of p53 and phospho-p53 in siBubR1-transfected HeLa cells treated with Cmpd-A and ispinesib. Cells were collected $48 \mathrm{~h}$ after drug treatment. (f) Anti-proliferative effect of Cmpd-A with or without siBubR1 in p53-wild-type and p53-knockout HCT116 cells. Twenty-four hours after siRNA treatment, the cells were treated with Cmpd-A at the indicated concentrations. Cells were collected on day 3 after drug treatment for the ATP assay. Relative ATP levels were calculated based on luminescence in comparison with the luminescence value for $0 \mathrm{nM}$ treatment. The line plots represent mean \pm s.d. $(n=3)$. Statistical analysis was performed using Student's $t$-test. Differences were considered significant at $P \leq 0.05\left({ }^{\star}\right)$ and $P \leq 0.01\left({ }^{\star *}\right)$.

kidney carcinoma cells) exhibited lower BubR1 expression than other cancer cell lines (Fig. 8a). FACS analysis of Caki-1 cells after Cmpd-A treatment revealed that the number of $\mathrm{pHH} 3$-positive cells was slightly increased $24 \mathrm{~h}$ after treatment $(6.7 \%$ with Cmpd-A and $2.0 \%$ with DMSO). However, the numbers decreased $48 \mathrm{~h}$ after treatment $(1.5 \%$ with Cmpd-A and $1.6 \%$ with
DMSO; Supplementary Fig. 17a). These data indicate that although the SAC machinery of Caki-1 cells is transiently activated in response to CENP-E inhibition, SAC activity is eventually reduced, allowing the cells to be released from prolonged mitotic arrest induced by mitotic slippage. Similar to siBubR1-transfected HeLa cells, Caki-1 cells treated with Cmpd-A 
a

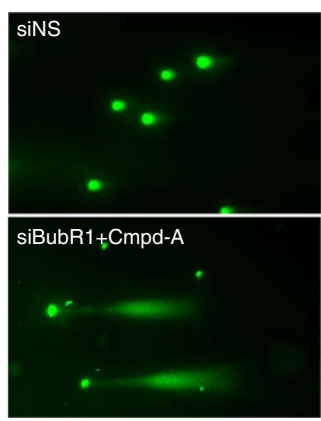

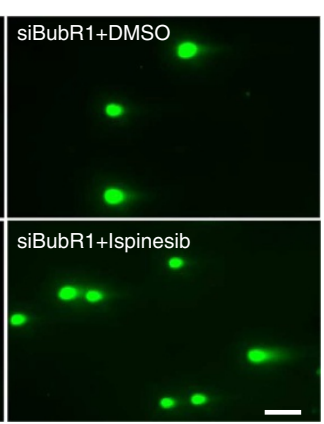

C

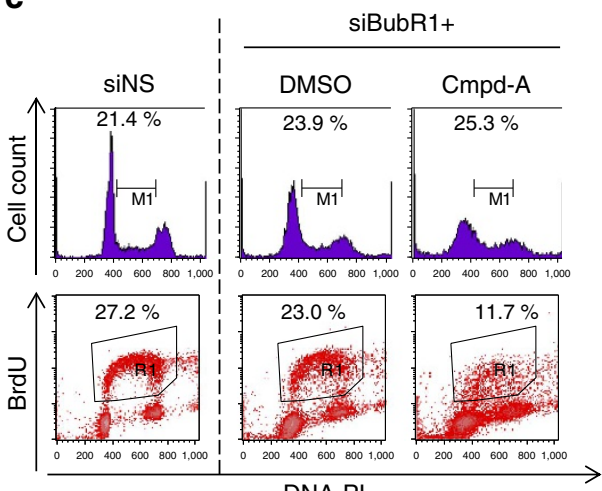

b

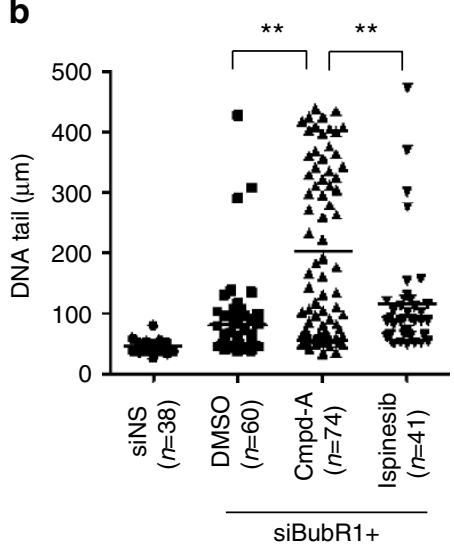

d

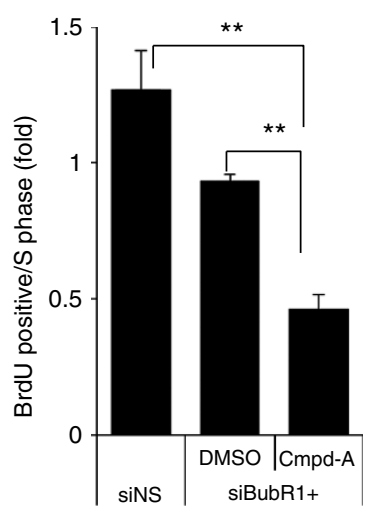

e

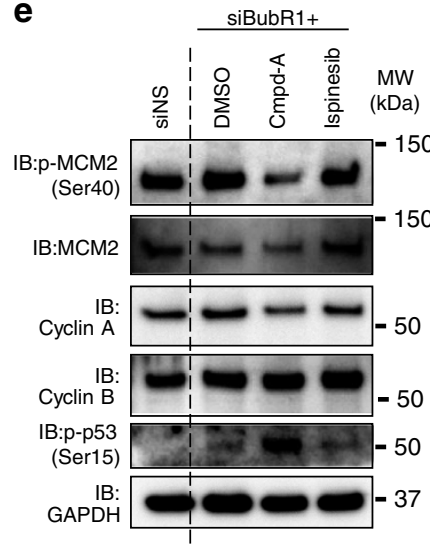

f
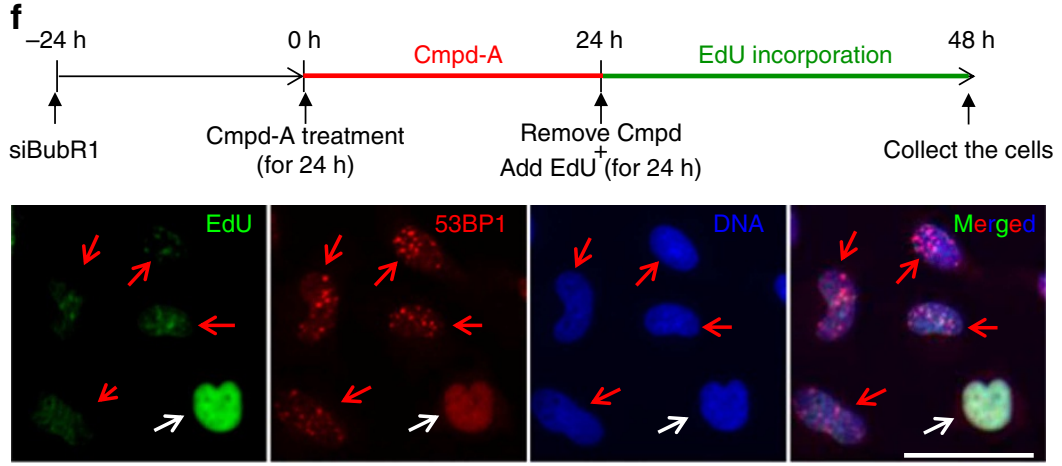

Figure 5 | CENP-E inhibition causes replication stress-mediated DSBs under SAC-impaired conditions. (a) Representative images of the neutral comet assay in siBubR1 + Cmpd-A-treated cells. Twenty-four hours after siRNA treatment, the cells were treated with DMSO, Cmpd-A (200 nM) or ispinesib $(10 \mathrm{nM})$ for $72 \mathrm{~h}$. The white bar indicates $100 \mu \mathrm{m}$. (b) Quantification of DNA tails in the neutral comet assay. The length of DNA tails in microscopy images was quantified by AxioVision. Statistical analysis was performed using Student's $t$-test. Differences were considered significant at $P \leq 0.01\left({ }^{\star \star}\right)$. (c) Cell cycle analysis in siBubR1 + Cmpd-A-treated cells. Twenty-four hours after siRNA treatment, the cells were treated with DMSO, Cmpd-A (200 nM) or ispinesib $(10 \mathrm{nM})$ for $72 \mathrm{~h}$. BrdU was incorporated into the drug-treated cells for $15 \mathrm{~min}$, and then the cells were collected for FACS analysis. Representative data are shown. (d) Replication activity in the S phase in siBubR1 $+\mathrm{Cmpd}$-A-treated cells. BrdU-positive cells (R1) were normalized to the $\mathrm{S}$ phase cells (M1). Data are presented as mean \pm s.d. $(n=3)$.Statistical analysis was performed using Student's $t$-test. Differences were considered significant at $P \leq 0.05\left(^{\star}\right)$ and $P \leq 0.01\left(^{\star \star}\right)$. (e) Immunoblotting of replication-regulating and $\mathrm{G} 2$ phase proteins in siBubR1 + Cmpd-A-treated cells. Cells were collected $72 \mathrm{~h}$ after drug treatment. (f) Correlation between 53BP1 foci formation and EdU incorporation. Schematics of the experiments are shown (above the panels). Red and white arrows indicate 53BP1 foci-positive and 53BP1 foci-negative cells, respectively. The white bar indicates $50 \mu \mathrm{m}$.

exhibited growth suppression (Fig. 8b, black line) accompanied by $\mathrm{p} 53$ accumulation and phosphorylation, p21 induction and increased $\gamma \mathrm{H} 2 \mathrm{AX}$ expression (Fig. 8b,c and Supplementary Fig. 17b). Treatment with Cmpd-A also induced time-dependent accumulation of p53, phospho-p53 and p21 in SAC-defective U87MG cells (Supplementary Fig. 17c), which exhibit less mitotic accumulation in response to colcemid (a microtubule depolymerizer). Immunostaining of the sections of a Caki-1 xenograft model revealed more p53-positive cells in Cmpd-Atreated tumours than in vehicle-treated tumours (Fig. 8d). Furthermore, Cmpd-A (at a concentration of $100 \mathrm{mg} \mathrm{kg}^{-1}$, administered three times (at 0,8 and $24 \mathrm{~h}$ ) on the first day of the study) displayed a potent antitumour effect (tumour growth inhibition $(\mathrm{T} / \mathrm{C})=16 \%$ on day 14$)$ without significant body weight loss (Fig. 8e and Supplementary Fig. 17d). Thus, although certain cancer cells such as Caki-1 and U87MG cells 
a

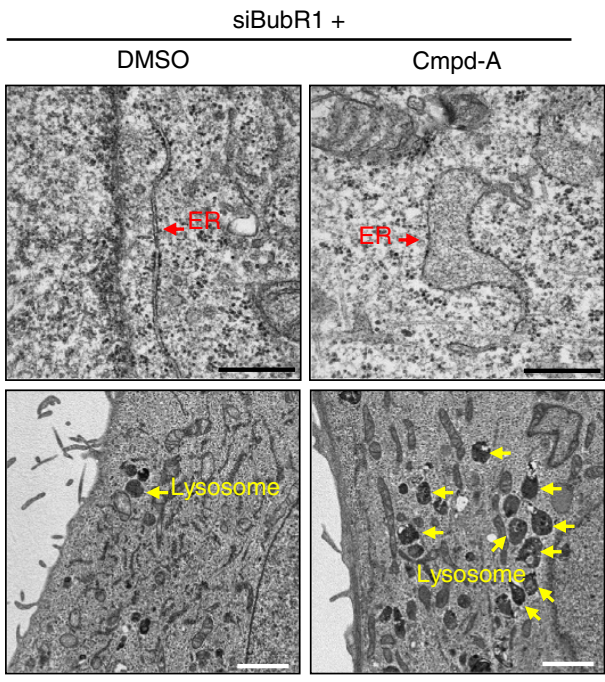

C

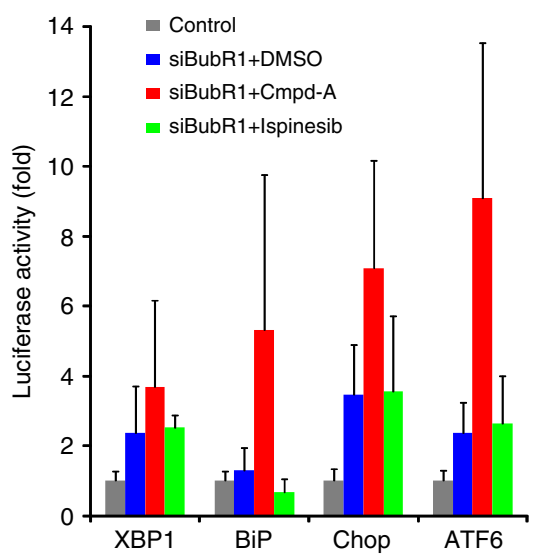

b

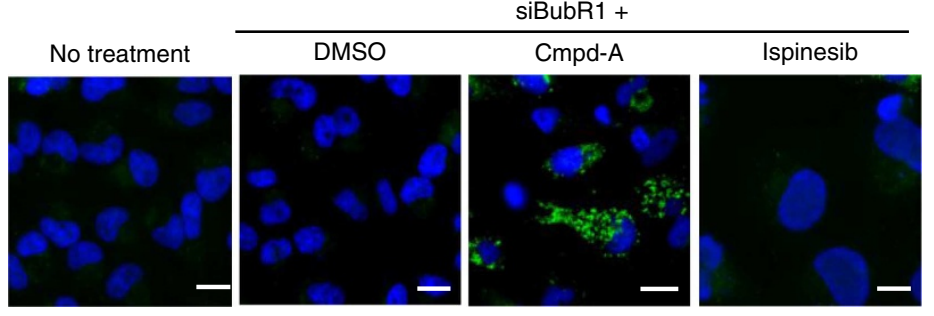

d

$\frac{\text { Pst-I digestion }}{\text { siBubR1+ }} \quad \frac{\text { No digestion }}{\text { siBubR1+ }}$

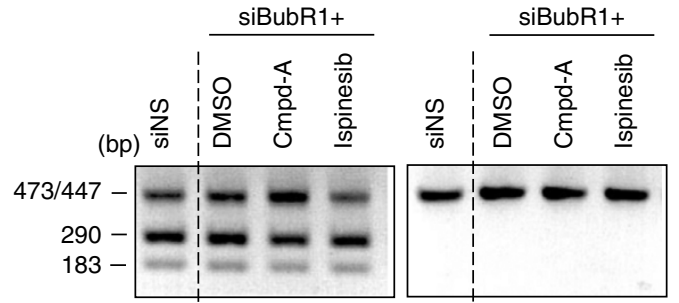

Figure 6 | Aneuploidy-associated proteotoxic stress is accompanied by p53 accumulation after mitotic slippage. (a) Electron microscopy images of the ER (upper panels, red arrows) and lysosome (lower panels, yellow arrows) in siBubR1+Cmpd-A-treated HeLa cells. Twenty-four hours after siRNA treatment, the cells were treated with Cmpd-A $(200 \mathrm{nM})$ or DMSO for $48 \mathrm{~h}$. Black and white bars indicate $500 \mathrm{~nm}$ and $2 \mu \mathrm{m}$, respectively. (b) Immunofluorescence of LC3B in siBubR1 + DMSO-, siBubR1 + Cmpd-A- and siBubR1+ ispinesib-treated HeLa cells. White bars indicate $20 \mu \mathrm{m}$. (c) Transcriptional reporter activities of XBP1, BiP, Chop and ATF6 in siBubR1 + Cmpd-A-treated HeLa cells. Transfection with siRNA was performed 24h before drug treatment, and the cells were treated with DMSO, Cmpd-A ( $200 \mathrm{nM})$ or ispinesib (10 nM) for $72 \mathrm{~h}$. Relative luciferase activities were calculated based on the luminescence values in comparison with siNS-transfected cells. Data are presented as mean \pm s.d. $(n=3$ ). (d) XBP1 splicing assay. cDNA was treated with (left) or without (right) Pst-I. The undigested upper band (473/447 bp) and the digested lower bands (290 and $183 \mathrm{bp)}$ represent spliced and unspliced XBP1, respectively.

spontaneously downregulate SAC proteins to attenuate the SAC machinery, CENP-E inhibition exerts anti-proliferative effects on these SAC-impaired cancer cells by activating p53 signalling pathways.

Finally, we examined BubR1 expression in primary tumours using immunohistochemistry (IHC; Fig. 9a and Supplementary Fig. 18a). IHC revealed low BubR1 expression in 13 of the 20 primary pancreatic tumour samples and 44 of the 70 tissue samples from other tumours (Fig. 9b). These results demonstrated reduced BubR1 expression in a considerable proportion of a broad spectrum of primary tumours, although this protein was upregulated in most cancer cell lines (Fig. 8a). Furthermore, $39.4 \%$ of the primary tumours exhibited low expression of both
BubR1 and p53 (Supplementary Fig. 18). Given that wild-type p53 tumours are closely associated with low p53 expression, it is possible that BubR1-depleted primary tumours carry the wild-type p53 gene. Although the SAC activity of these BubR1-depleted primary tumours remains to be elucidated, it is possible that a subset of these tumours, similar to Caki-1 cells, could attenuate the SAC machinery to escape mitotic death under mitotic inhibition.

\section{Discussion}

In this study, we assessed the potential of CENP-E and Eg5 as cancer therapeutic targets using siRNAs and chemical inhibitors. Detailed mechanistic analyses revealed that CENP-E inhibition, 
a
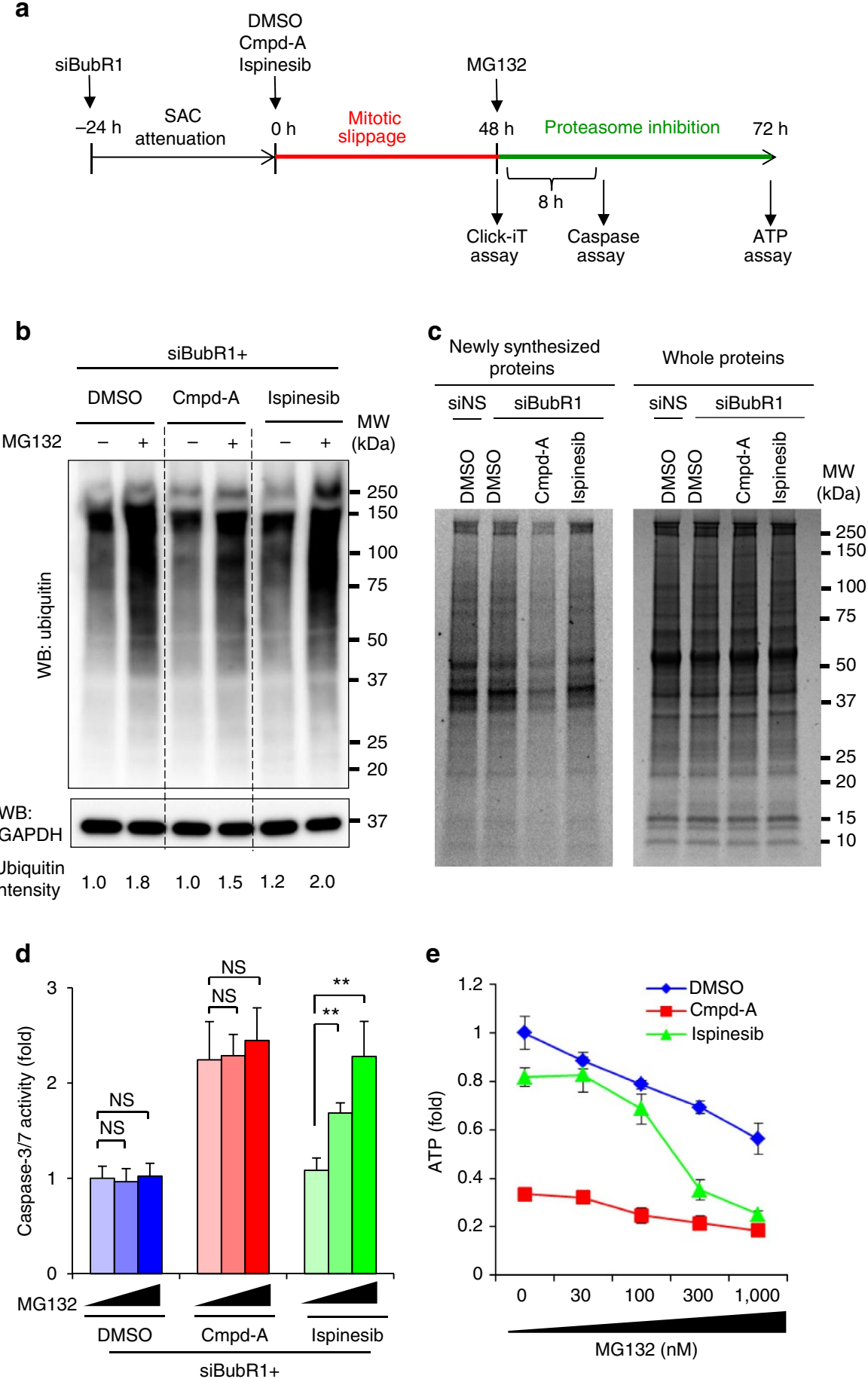

Figure 7 | siBubR1 + Cmpd-A aneuploid cells decrease global protein translation to reduce the response to the proteasome inhibitor MG132.

(a) Schematics of the MG132 treatment experiments. (b) Global protein ubiquitination by MG132 in siBubR1+DMSO-, siBubR1+Cmpd-A- and siBubR1 + ispinesib-treated HeLa cells. (c) Newly synthesized protein in siBubR1 + DMSO-, siBubR1 + Cmpd-A- and siBubR1 + ispinesib-treated HeLa cells. Left and right gels show newly synthesized proteins detected by the Click-iT assay (Life Technologies) and all proteins detected by CBB staining as a loading control, respectively. (d) Effect of MG132 on caspase-3/7 activity in siBubR1 + DMSO-, siBubR1 + Cmpd-A- and siBubR1+ispinesib-treated HeLa cells. Blue, red and green bars indicate siBubR1 + DMSO-, siBubR1 + Cmpd-A- and siBubR1 + ispinesib-treated HeLa cells, respectively. Relative caspase-3/7 activities were calculated in comparison with the activities in siBubR1+DMSO-treated cells. Statistical analysis was performed using Student's $t$-test. Differences were considered significant at $P \leq 0.05\left(^{(}\right)$and $P \leq 0.01\left(^{(*}\right)$. (e). Effect of MG132 on cell proliferation in siBubR1+DMSO-, siBubR1+Cmpd-Aand siBubR1 + ispinesib-treated HeLa cells. Blue, red and green lines indicate siBubR1 + DMSO-, siBubR1 + Cmpd-A- and siBubR1 + ispinesib-treated HeLa cells, respectively. Relative ATP levels were calculated in comparison with those in siBubR1 + DMSO-treated cells. Relative ATP levels were calculated based on luminescence in comparison with the luminescence value for 0 -nM treatment. The line plots represent mean \pm s.d. $(n=3)$. 
a

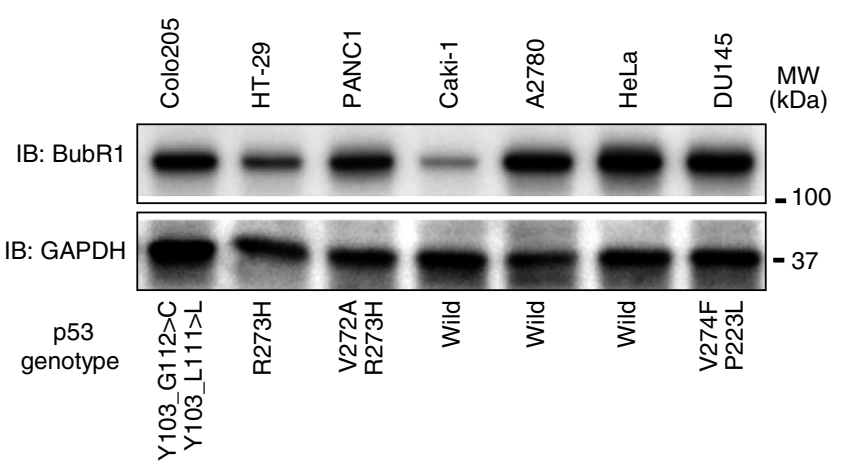

C

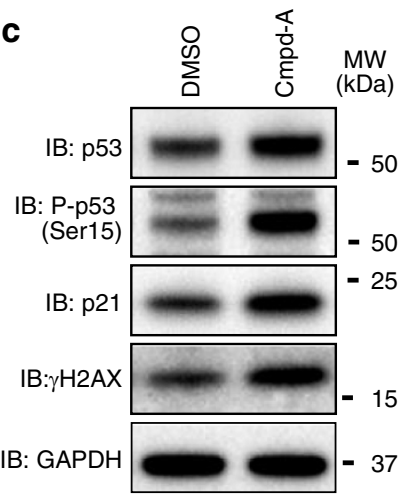

b
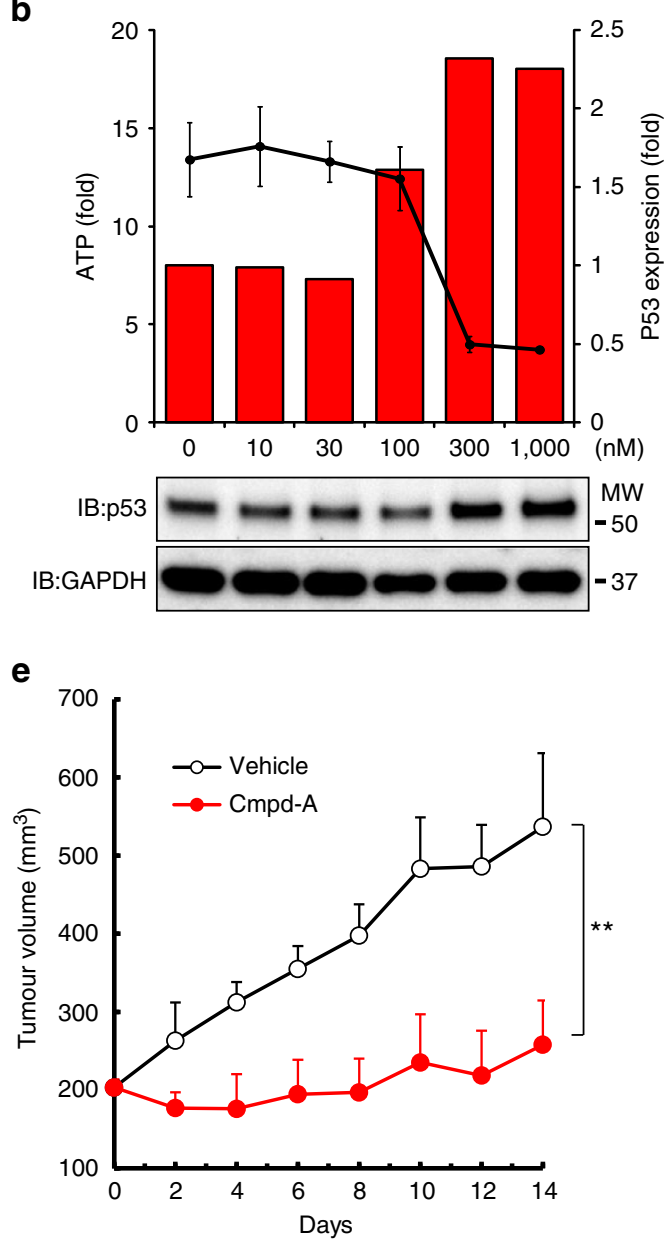

d

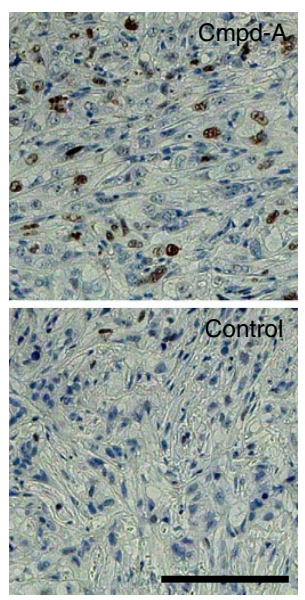

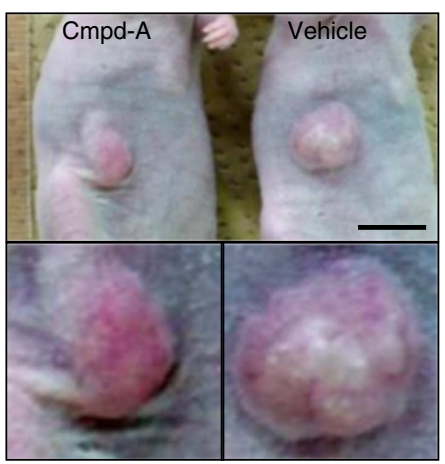

Figure 8 | Cmpd-A increases tumour resistance in SAC-impaired Caki-1 cells in vitro and in vivo. (a) BubR1 downregulation in SAC-impaired Caki-1 cells. The COSMIC database was used for p53 genotyping of each cell line. (b) Anti-proliferative activity of Cmpd-A (black line) and p53 accumulation (red bars) in Caki-1. p53 levels were quantified using immunoblotting results. The line plots represent mean \pm s.d. $(n=3)$. (c) p53 accumulation and DSBs in Caki-1 cells after $\mathrm{Cmpd}-\mathrm{A}$ treatment. Cells were collected $48 \mathrm{~h}$ after treatment. (d) $\mathrm{lHC}$ of p53 in Caki- 1 xenografts. Mice were administered $100 \mathrm{mg} \mathrm{kg}{ }^{-1} \mathrm{Cmpd}-\mathrm{A}$ twice $(0$ and $8 \mathrm{~h}$ ). The tumours were collected $24 \mathrm{~h}$ after the first administration. The white bar indicates $100 \mu \mathrm{m}$. (e) The antitumour efficacy of $\mathrm{Cmpd}-\mathrm{A}$ in the Caki-1 xenograft models (right). The line plots represent mean \pm s.d. $(n=5)$. The representative xenografts on day 8 after administration are shown (left). The black bar indicates $1 \mathrm{~cm}$.

but not Eg5 inhibition, induces post-mitotic effects through a unique mechanism of action involving aneuploidy-mediated p53dependent post-mitotic apoptosis. We also demonstrated that aneuploidy generates both replication stress-mediated DSBs and proteotoxic stress to activate DDR and UPR pathways (Fig. 9c). These integrated signalling pathways, in collaboration with SAC, play important roles in eliminating chromosome instability.
Antimitotic therapies that target microtubule dynamics, such as taxanes or vinca alkaloids, are widely used in the clinical treatment of cancer ${ }^{10}$. Following attempts to improve the therapeutic properties of these microtubule inhibitors 46,47 , nonstructural components of microtubules that are key components of mitosis have recently attracted attention. Several smallmolecule inhibitors that induce mitotic aberrations are 
a

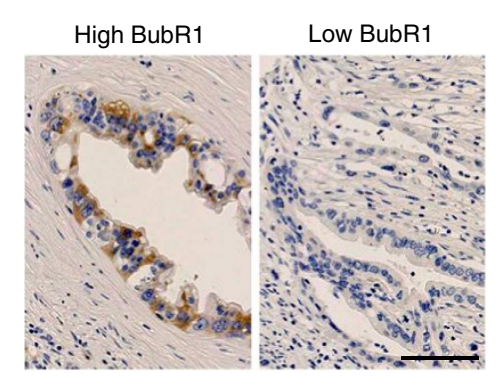

b

\begin{tabular}{lcc} 
& \multicolumn{2}{c}{ BubR1 expression } \\
\cline { 2 - 3 } Primary tumour tissues & High & Low \\
\hline Adrenal gland & 0 & 1 \\
Bladder, urinary & 1 & 1 \\
Breast & 1 & 2 \\
Bone & 1 & 1 \\
Brain, cerebellum & 0 & 1 \\
Oesophagus & 0 & 3 \\
Stomach & 1 & 2 \\
Intestine, small intestine & 0 & 1 \\
Intestine, colon & 1 & 1 \\
intestine, rectum & 1 & 1 \\
Kidney & 2 & 0 \\
Liver & 1 & 3 \\
Lung & 3 & 1 \\
Lymph node & 0 & 3 \\
Head and neck & 0 & 3 \\
Ovary & 2 & 0 \\
Pancreas & 7 & 13 \\
Prostate & 0 & 2 \\
Skin & 0 & 2 \\
Testis & 1 & 1 \\
Thyroid & 0 & 2 \\
Uterus & 4 & 0 \\
\hline Total $(N=70)$ & 26 & 44 \\
& $(37 \%)$ & $(63 \%)$
\end{tabular}

C

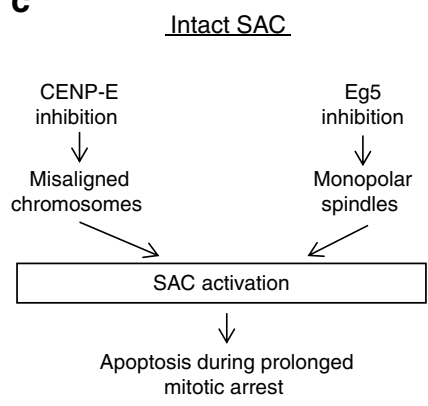

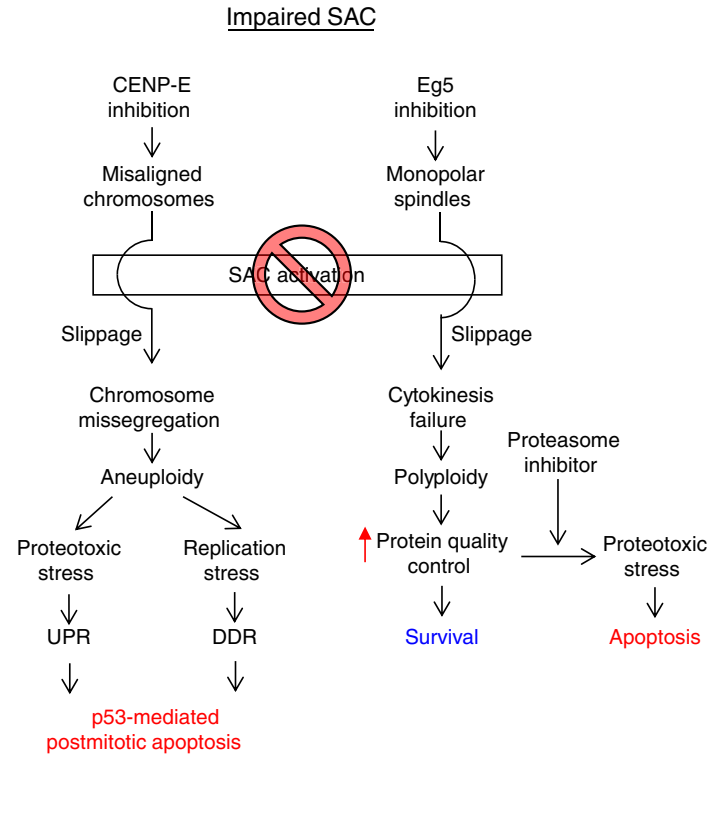

Figure 9 | IHC of BubR1 in primary tumour tissues. (a) IHC of BubR1 in primary pancreatic tumours. The representative images of high (left panel) and low expression (right panel) of BubR1 are shown. The black bar indicates $100 \mu \mathrm{m}$. (b) Summary of IHC of BubR1 in 70 different tumour tissues. The tissue microarrays were purchased from BioChain Institute Inc. (c) Schematics of aneuploidy-mediated apoptosis after mitotic slippage.

undergoing clinical trials ${ }^{10,18,25,32}$. Accumulated evidence suggests that the SAC machinery is responsible for the sensitivity of cancer cells to antimitotic agents ${ }^{12-15}$, although the SAC mechanism is attenuated in a broad spectrum of primary tumours (Figs 8 and 9) ${ }^{48-50}$. In this study, we demonstrated that aneuploidy caused by CENP-E inhibition has potent post-mitotic anti-proliferative p53-dependent effects on SAC-impaired cells. Polyploidy caused by Eg5 inhibition does not produce this effect. Our results are in agreement with those of some recent genetic studies reporting elevated cell death and decreased tumour formation in CENP-E $\mathrm{E}^{+1-} \mathrm{Mad} 2+1-$ doubleheterozygous mice ${ }^{51}$. Thus, CENP-E inhibition may be effective against cancers refractory to other mitotic inhibitors, such as SAC-attenuated cancers, and it could potentially expand the therapeutic window for cancer drugs.

Although various mitotic inhibitors are being developed, the underlying mechanism of action remains unclear. First, the cellular events associated with reduced proliferation after mitotic slippage are not well understood. One hypothesis proposes that the microtubule-dependent post-mitotic checkpoint is responsible for cell cycle arrest after mitotic slippage ${ }^{52,53}$. Another controversial hypothesis proposes that prolonged mitotic arrest itself, but not the microtubule alternation checkpoint, is responsible for this phenomenon ${ }^{54,55}$. We propose an alternative hypothesis. In our model, aneuploidy-associated replication stress and proteotoxic stress also contribute to antiproliferation in cancer cells after mitotic slippage. We revealed that siCENP-E + siBubR1 did not cause prolonged mitosis or aberrant microtubule structure formation.

Second, the mechanism by which mitotic aberration generates DNA damage is unclear. One possibility is that DNA damage accumulates during prolonged mitotic arrest ${ }^{56,57}$. However, in our model, prolonged mitotic arrest was not induced by either siBubR1 + siCENP-E or siBubR1 + Cmpd-A; thus, aneuploidyinduced DSBs could be generated independently of the duration of mitotic arrest. Another study proposed that the chromosome bridge during cytokinesis generates DSBs and activates DDR pathways $^{58}$. In that study, $\gamma \mathrm{H} 2 \mathrm{AX}$ and 53BP1 foci were restricted 
to DNA positioned in or close to the cleavage furrow of cytokinesis, and most cells $(\sim 80 \%)$ accumulated 53BP1 foci within $2 \mathrm{~h}$ after chromosome missegregation. In our model, DSBs were mainly generated between 24 and $48 \mathrm{~h}$ after mitotic slippage. In addition, larger numbers of 53BP1 foci were observed compared with the previous study (Fig. 5c). Thus, DSBs detected in our models appear to be caused by chronic chromosome instability rather than by chromosome breaks in a lagging chromosome. A third hypothesis proposes that micronuclei produced by lagging chromosomes generate DNA damage with defective and asynchronous DNA replication and aberrant DDR protein recruitment ${ }^{59}$. However, in our models, 53BP1 foci were mainly observed at primary nuclei rather than micronuclei (Fig. 5c). Our results demonstrate that chronic DNA replication stress after chromosome missegregation also contributes to aneuploidy-associated DSBs. In yeast, aneuploid cells display increased chromosome loss and mitotic recombination as well as defective DNA damage repair ${ }^{60}$. With replication stress during the $S$ phase, the defects in DNA repair systems may increase DSB accumulation in aneuploid cells.

Third, it is unclear which molecules or pathways monitor chromosome missegregation after mitotic slippage and how these monitoring systems could exclude the aneuploid cells. Our study demonstrated that p53 activation, accompanied by aneuploidymediated DSBs and proteotoxic stress, is responsible for post-mitotic apoptosis excluding the aneuploid cells. Recent studies have demonstrated functional relationships between protein quality control systems and chromosome instability in aneuploid cells ${ }^{38,39,61,62}$. These results suggest that rapid changes in chromosome copy numbers could alter cellular protein homeostasis markedly. Expectedly, our model also detected phenotypes of substantial proteotoxic stress in siBubR1 + Cmpd-A-treated aneuploid cells: pathological changes of ER, aggresome formation, transcriptional activation of UPR genes, spliced form of XBP1 and autophagic vesicle-associated LC3B-II (Fig. 6). Although several studies have demonstrated that ER stress could regulate p53 pathways positively or negatively, the mechanisms underlying this phenomenon remain unclear ${ }^{63-66}$. Our study revealed that p53-mediated post-mitotic apoptosis is accompanied by DDR and UPR activation (Fig. 6). UPR, in collaboration with DDR, may play an important role in eliminating aneuploid cells after mitotic slippage in a p53dependent manner. The levels of UPR activation and autophagy were lower in polyploid cells than in aneuploid cells. Polyploid cells were more sensitive to proteasome inhibition, although the extent of ubiquitination of global proteins and the levels of new protein synthesis were similar to those in the control cells (Fig. 7). Other polyploidy-specific aspects, such as enhanced cellular metabolism 9,67 , may increase the sensitivity of these cells to proteotoxic stress.

In conclusion, we revealed that under SAC-defective conditions, aneuploidy caused by CENP-E inhibition, but not polyploidy induced by Eg5 inhibition, triggers p53 activation after mitotic slippage, resulting in post-mitotic suppression of proliferation. Furthermore, aneuploidy-associated DNA damage and proteotoxic stress are accompanied by $\mathrm{p} 53$-mediated postmitotic apoptosis in SAC-attenuated cancer cells. Our study provides some new insights into the molecular mechanisms of cancer cell elimination by massive chromosome instability caused by mitotic inhibitors. It also demonstrates the therapeutic potential of CENP-E inhibitors as cancer drugs.

\section{Methods}

Compounds. Cmpd-A was synthesized by Takeda Pharmaceutical Company Ltd (Supplementary Methods). Ku55933 and ispinesib were purchased from Wako Pure Chemical Industries Ltd., and Selleck Chemicals, LLC, respectively.
Cell growth and caspase-3/7 assays. Cell growth was evaluated by measuring protein amounts by crystal violet staining or intracellular ATP concentrations using the CellTiter-Glo luminescent cell viability assay (Promega Corp., Madison, WI, USA). Caspase-3/7 activity was evaluated using a Caspase-3/7-Glo luminescent kit (Promega). Absorbance and chemical luminescence were measured using a microplate reader.

Immunofluorescence assay. Cells were fixed for $15 \mathrm{~min}$ in PBS-buffered $4 \%$ paraformaldehyde, followed by permeablization for $5 \mathrm{~min}$ in Triton-X buffer ${ }^{68}$. The following antibodies were used at a concentration of $1-2 \mu \mathrm{g} \mathrm{ml}^{-1}$ for immunofluorescence assays: anti-CENP-B (sc22788; Santa Cruz Biotechnology), anti-BubR1 (612503), anti- $\alpha$-tubulin (T9026; Sigma-Aldrich), anti-p53 (sc126; Santa Cruz Biotechnology), anti-53BP1 (sc22760; Santa Cruz Biotechnology), antip62 (sc28359; Santa Cruz Biotechnology) and anti-LC3B (3868; Cell Signaling Technology). Images were captured with a Plan-APOCHROMAT $\times 100$ oil lens under an Axiovert 200M microscope (Carl Zeiss).

In vivo efficacy studies. Caki- 1 cells $\left(3-5 \times 10^{6}\right.$ cells per mouse) were xenografted into $\mathrm{BALB} / \mathrm{c}$ nude mice (7 weeks old, female). Mice bearing tumours (100$250 \mathrm{~mm}^{3}$ ) were selected and randomly categorized into vehicle and Cmpd-A groups ( 5 mice per group). The xenografted mice were intraperitoneally administered $100 \mathrm{mg} \mathrm{kg}^{-1} \mathrm{Cmpd}-\mathrm{A} 3$ times (at 0,8 and $24 \mathrm{~h}$ ) on the first day of the study. T/C (\%) was calculated using the following formula:

$\mathrm{T} / \mathrm{C}(\%)=((\mathrm{Cmpd}-\mathrm{A}$ tumour volume $-\mathrm{Cmpd}-\mathrm{A}$ tumour volume on day 0$) /$ (vehicle tumour volume - vehicle tumour volume on day 0$)$ ) $\times 100$

All in vivo procedures were performed in accordance with the protocols approved by the Takeda Experimental Animal Care and Use Committee.

Time-lapse imaging and analysis. HeLa cells were cultured in 24-well culture plates with glass bottoms (MatTek Corp., Ashland, MA, USA) and transfected with siRNA oligos targeting the indicated genes. Phase-contrast images were captured every $10 \mathrm{~min}$ for $24 \mathrm{~h}$ (12-36 h after transfection). Cells were imaged using an Axiovert 200M microscope equipped with EC Plan NEOFLUAR $\times 20$ lens (Carl Zeiss). Images were acquired and processed using AxioVision 4.5 software (Carl Zeiss). The images were converted to JPEG format and exported to Adobe Photoshop.

FACS analysis. Cells fixed with $70 \%$ ethanol were incubated for $30 \mathrm{~min}$ in PBS containing $2 \%$ fetal bovine serum, $100 \mu \mathrm{g} \mathrm{ml}^{-1}$ RNase A (Sigma-Aldrich) and $1 \mu \mathrm{g} \mathrm{ml}^{-1}$ Alexa Fluor 488-conjugated $\mathrm{pHH} 3$ antibodies (1:50 dilution, Cat\# 3465, Cell Signaling Technology, Danvers, MA, USA). After washing, the cells were incubated in $50 \mu \mathrm{g} \mathrm{ml}^{-1}$ propidium iodide (Sigma-Aldrich). Ten thousand cells were analysed using FACSCalibur (Becton-Dickinson, Franklin Lakes, NJ, USA).

Detection of newly synthesized proteins and immunoblotting. Newly synthesized proteins were detected using the Click-iT reagent kit (Life Technologies) according to the manufacturer's protocol. In brief, siBubR1-transfected HeLa cells were treated with the indicated compounds for $48 \mathrm{~h}$ and then incubated for $30 \mathrm{~min}$ in methionine-free culture medium. Then, the cells were cultured for $4 \mathrm{~h}$ in the presence of $50 \mu \mathrm{M}$ L-Azidohomoalanine (Life Technologies). To label the newly synthesized proteins, click reactions were performed using $25-\mu$ g aliquots of proteins from each cell lysate. Following SDS-polyacrylamide gel electrophoresis, the proteins were detected using LAS-3000 (FujiFilm). Uncropped immunoblots are shown in Supplementary Figs 19 and 20.

\section{References}

1. Musacchio, A. \& Salmon, E. D. The spindle-assembly checkpoint in space and time. Nat. Rev. Mol. Cell Biol. 8, 379-393 (2007).

2. Wassmann, K. \& Benezra, R. Mitotic checkpoints: from yeast to cancer. Curr. Opin. Genet. Dev. 11, 83-90 (2001).

3. Cleveland, D. W., Mao, Y. \& Sullivan, K. F. Centromeres and kinetochores: from epigenetics to mitotic checkpoint signaling. Cell 112, 407-421 (2003).

4. Kulukian, A., Han, J. S. \& Cleveland, D. W. Unattached kinetochores catalyze production of an anaphase inhibitor that requires a Mad2 template to prime Cdc20 for BubR1 binding. Dev. Cell 16, 105-117 (2009).

5. Topham, C. H. \& Taylor, S. S. Mitosis and apoptosis: how is the balance set? Curr. Opin. Cell Biol. 25, 780-785 (2013).

6. Bakhoum, S. F. \& Compton, D. A. Chromosomal instability and cancer: a complex relationship with therapeutic potential. J. Clin. Invest. 122, 1138-1143 (2012).

7. Fodde, R. et al. Mutations in the APC tumour suppressor gene cause chromosomal instability. Nat. Cell Biol. 3, 433-438 (2001).

8. Kaplan, K. B. et al. A role for the adenomatous polyposis coli protein in chromosome segregation. Nat. Cell Biol. 3, 429-432 (2001).

9. Davoli, T. \& de Lange, T. The causes and consequences of polyploidy in normal development and cancer. Annu. Rev. Cell Dev. Biol. 27, 585-610 (2011). 
10. Jackson, J. R., Patrick, D. R., Dar, M. M. \& Huang, P. S. Targeted anti-mitotic therapies: can we improve on tubulin agents? Nat. Rev. Cancer 7, 107-117 (2007).

11. Gascoigne, K. E. \& Taylor, S. S. Cancer cells display profound intra- and interline variation following prolonged exposure to antimitotic drugs. Cancer Cell 14, 111-122 (2008).

12. Sudo, T., Nitta, M., Saya, H. \& Ueno, N. T. Dependence of paclitaxel sensitivity on a functional spindle assembly checkpoint. Cancer Res. 64, 2502-2508 (2004).

13. Anand, S., Penrhyn-Lowe, S. \& Venkitaraman, A. R. AURORA-A amplification overrides the mitotic spindle assembly checkpoint, inducing resistance to Taxol. Cancer Cell 3, 51-62 (2003).

14. Huang, H. C., Shi, J., Orth, J. D. \& Mitchison, T. J. Evidence that mitotic exit is a better cancer therapeutic target than spindle assembly. Cancer Cell 16, 347-358 (2009).

15. Fu, Y. et al. Weakened spindle checkpoint with reduced BubR1 expression in paclitaxel-resistant ovarian carcinoma cell line SKOV3-TR30. Gynecol. Oncol. 105, 66-73 (2007)

16. Swanton, C. et al. Regulators of mitotic arrest and ceramide metabolism are determinants of sensitivity to paclitaxel and other chemotherapeutic drugs. Cancer Cell 11, 498-512 (2007).

17. Miki, H., Okada, Y. \& Hirokawa, N. Analysis of the kinesin superfamily: insights into structure and function. Trends Cell Biol. 15, 467-476 (2005).

18. Tao, W. et al. Induction of apoptosis by an inhibitor of the mitotic kinesin KSP requires both activation of the spindle assembly checkpoint and mitotic slippage. Cancer Cell 8, 49-59 (2005).

19. Luo, L. et al. ATP-competitive inhibitors of the mitotic kinesin KSP that function via an allosteric mechanism. Nat. Chem. Biol. 3, 722-726 (2007)

20. Lad, L. et al. Mechanism of inhibition of human KSP by ispinesib. Biochemistry 47, 3576-3585 (2008).

21. Yen, T. J., Li, G., Schaar, B. T., Szilak, I. \& Cleveland, D. W. CENP-E is a putative kinetochore motor that accumulates just before mitosis. Nature 359, 536-539 (1992)

22. Yao, X., Anderson, K. L. \& Cleveland, D. W. The microtubule-dependent motor centromere-associated protein E (CENP-E) is an integral component of kinetochore corona fibers that link centromeres to spindle microtubules. J. Cell Biol. 139, 435-447 (1997).

23. Wood, K. W., Sakowicz, R., Goldstein, L. S. \& Cleveland, D. W. CENP-E is a plus end-directed kinetochore motor required for metaphase chromosome alignment. Cell 91, 357-366 (1997).

24. Barisic, M., Aguiar, P., Geley, S. \& Maiato, H. Kinetochore motors drive congression of peripheral polar chromosomes by overcoming random armejection forces. Nat. Cell Biol. 16, 1249-1256 (2014).

25. Wood, K. W. et al. Antitumor activity of an allosteric inhibitor of centromereassociated protein-E. Proc. Natl Acad. Sci. USA 107, 5839-5844 (2010).

26. Schaar, B. T., Chan, G. K., Maddox, P., Salmon, E. D. \& Yen, T. J. CENP-E function at kinetochores is essential for chromosome alignment. J. Cell Biol. 139, 1373-1382 (1997).

27. Yao, X., Abrieu, A., Zheng, Y., Sullivan, K. F. \& Cleveland, D. W. CENP-E forms a link between attachment of spindle microtubules to kinetochores and the mitotic checkpoint. Nat. Cell Biol. 2, 484-491 (2000).

28. Putkey, F. R. et al. Unstable kinetochore-microtubule capture and chromosomal instability following deletion of CENP-E. Dev. Cell 3, 351-365 (2002).

29. Mao, Y., Desai, A. \& Cleveland, D. W. Microtubule capture by CENP-E silences BubR1-dependent mitotic checkpoint signaling. J. Cell Biol. 170, 873-880 (2005).

30. Weaver, B. A. \& Cleveland, D. W. Aneuploidy: instigator and inhibitor of tumorigenesis. Cancer Res. 67, 10103-10105 (2007).

31. Chung, V. et al. First-time-in-human study of GSK923295, a novel antimitotic inhibitor of centromere-associated protein E (CENP-E), in patients with refractory cancer. Cancer Chemother. Pharmacol. 69, 733-741 (2011).

32. Rath, O. \& Kozielski, F. Kinesins and cancer. Nat. Rev. Cancer 12, 527-539 (2012).

33. Kung, P. P. et al. Chemogenetic evaluation of the mitotic kinesin CENP-E reveals a critical role in triple-negative breast cancer. Mol. Cancer Ther. 13, 2104-2115 (2014).

34. Kapitein, L. C. et al. The bipolar mitotic kinesin Eg5 moves on both microtubules that it crosslinks. Nature 435, 114-118 (2005).

35. Gruneberg, U., Neef, R., Honda, R., Nigg, E. A. \& Barr, F. A. Relocation of Aurora $\mathrm{B}$ from centromeres to the central spindle at the metaphase to anaphase transition requires MKlp2. J. Cell Biol. 166, 167-172 (2004).

36. Charrier, J. D. et al. Discovery of potent and selective inhibitors of ataxia telangiectasia mutated and Rad3 related (ATR) protein kinase as potential anticancer agents. J. Med. Chem. 54, 2320-2330 (2011).

37. Reaper, P. M. et al. Selective killing of ATM- or p53-deficient cancer cells through inhibition of ATR. Nat. Chem. Biol. 7, 428-430 (2011).

38. Torres, E. M. et al. Identification of aneuploidy-tolerating mutations. Cell 143, $71-83$ (2010).
39. Oromendia, A. B., Dodgson, S. E. \& Amon, A. Aneuploidy causes proteotoxic stress in yeast. Genes Dev. 26, 2696-2708 (2012).

40. Bjorkoy, G. et al. p62/SQSTM1 forms protein aggregates degraded by autophagy and has a protective effect on huntingtin-induced cell death. J. Cell Biol. 171, 603-614 (2005).

41. Pankiv, S. et al. p62/SQSTM1 binds directly to Atg8/LC3 to facilitate degradation of ubiquitinated protein aggregates by autophagy. J. Biol. Chem. 282, 24131-24145 (2007).

42. Dorr, J. R. et al. Synthetic lethal metabolic targeting of cellular senescence in cancer therapy. Nature 501, 421-425 (2013).

43. He, C. \& Klionsky, D. J. Regulation mechanisms and signaling pathways of autophagy. Annu. Rev. Genet. 43, 67-93 (2009).

44. Wang, S. \& Kaufman, R. J. The impact of the unfolded protein response on human disease. J. Cell Biol. 197, 857-867 (2012).

45. Calfon, M. et al. IRE1 couples endoplasmic reticulum load to secretory capacity by processing the XBP-1 mRNA. Nature 415, 92-96 (2002).

46. Jordan, M. A. \& Wilson, L. Microtubules as a target for anticancer drugs. Nat. Rev. Cancer 4, 253-265 (2004).

47. Orr, G. A., Verdier-Pinard, P., McDaid, H. \& Horwitz, S. B. Mechanisms of Taxol resistance related to microtubules. Oncogene 22, 7280-7295 (2003).

48. Shin, H. J. et al. Dual roles of human BubR1, a mitotic checkpoint kinase, in the monitoring of chromosomal instability. Cancer Cell 4, 483-497 (2003).

49. Kops, G. J., Weaver, B. A. \& Cleveland, D. W. On the road to cancer: aneuploidy and the mitotic checkpoint. Nat. Rev. Cancer 5, 773-785 (2005).

50. Weaver, B. A. \& Cleveland, D. W. Does aneuploidy cause cancer? Curr. Opin. Cell Biol. 18, 658-667 (2006).

51. Silk, A. D. et al. Chromosome missegregation rate predicts whether aneuploidy will promote or suppress tumors. Proc. Natl Acad. Sci. USA 110, E4134-E4141 (2013).

52. Lanni, J. S. \& Jacks, T. Characterization of the p53-dependent postmitotic checkpoint following spindle disruption. Mol. Cell. Biol. 18, 1055-1064 (1998)

53. Blajeski, A. L., Phan, V. A., Kottke, T. J. \& Kaufmann, S. H. G(1) and G(2) cellcycle arrest following microtubule depolymerization in human breast cancer cells. J. Clin. Invest. 110, 91-99 (2002).

54. Uetake, Y. \& Sluder, G. Cell-cycle progression without an intact microtuble cytoskeleton. Curr. Biol. 17, 2081-2086 (2007).

55. Uetake, Y. \& Sluder, G. Prolonged prometaphase blocks daughter cell proliferation despite normal completion of mitosis. Curr. Biol. 20, 1666-1671 (2010)

56. Dalton, W. B., Nandan, M. O., Moore, R. T. \& Yang, V. W. Human cancer cells commonly acquire DNA damage during mitotic arrest. Cancer Res. 67, 11487-11492 (2007).

57. Quignon, F. et al. Sustained mitotic block elicits DNA breaks: one-step alteration of ploidy and chromosome integrity in mammalian cells. Oncogene 26, 165-172 (2007).

58. Janssen, A., van der Burg, M., Szuhai, K., Kops, G. J. \& Medema, R. H. Chromosome segregation errors as a cause of DNA damage and structural chromosome aberrations. Science 333, 1895-1898 (2011).

59. Crasta, K. et al. DNA breaks and chromosome pulverization from errors in mitosis. Nature 482, 53-58 (2012).

60. Sheltzer, J. M. et al. Aneuploidy drives genomic instability in yeast. Science $\mathbf{3 3 3}$, 1026-1030 (2011).

61. Torres, E. M. et al. Effects of aneuploidy on cellular physiology and cell division in haploid yeast. Science 317, 916-924 (2007).

62. Tang, Y. C., Williams, B. R., Siegel, J. J. \& Amon, A. Identification of aneuploidy-selective antiproliferation compounds. Cell 144, 499-512 (2011).

63. Qu, L. et al. Endoplasmic reticulum stress induces p53 cytoplasmic localization and prevents p53-dependent apoptosis by a pathway involving glycogen synthase kinase-3beta. Genes Dev. 18, 261-277 (2004).

64. Pluquet, O., Qu, L. K., Baltzis, D. \& Koromilas, A. E. Endoplasmic reticulum stress accelerates p53 degradation by the cooperative actions of $\mathrm{Hdm} 2$ and glycogen synthase kinase 3beta. Mol. Cell. Biol. 25, 9392-9405 (2005).

65. Baltzis, D. et al. The eIF2alpha kinases PERK and PKR activate glycogen synthase kinase 3 to promote the proteasomal degradation of p53. J. Biol. Chem. 282, 31675-31687 (2007).

66. Li, J., Lee, B. \& Lee, A. S. Endoplasmic reticulum stress-induced apoptosis: multiple pathways and activation of p53-up-regulated modulator of apoptosis (PUMA) and NOXA by p53. J. Biol. Chem. 281, 7260-7270 (2006).

67. Lee, H. O., Davidson, J. M. \& Duronio, R. J. Endoreplication: polyploidy with purpose. Genes Dev. 23, 2461-2477 (2009).

68. Ohashi, A., Zdzienicka, M. Z., Chen, J. \& Couch, F. J., Fanconi anemia complementation group. D2 (FANCD2) functions independently of BRCA2and RAD51-associated homologous recombination in response to DNA damage. J. Biol. Chem. 280, 14877-14883 (2005)

\section{Acknowledgements}

We are grateful to Dr Akito Nakamura, Dr Yoshinori Ishikawa, Saomi Murai, Kazuhide Nakayama, Dr Shinsuke Araki, Dr Masato Yabuki, Dr Hiromichi Kimura, Sei Yoshida and Dr Toshiyuki Nomura for valuable comments on the manuscript. We also express 
our appreciation for the technical supports of Mr Motoo Iida and Ms Yasumi Kumagai in NMR spectroscopic analysis. This research was supported by Takeda Pharmaceutical Company Ltd.

\section{Author contributions}

A.O. conceptualized the study. A.O., M.O (Ohori). and K.I. designed the experiments. A.O. and M.O (Okaniwa). wrote the manuscript. A.O., M.O (Ohori)., K.I. and T.N. performed the experiments. Y.N. performed the bioinformatics analysis of microarray data. D.M. generated the UPR reporter assay systems. T.H., M.O (Okaniwa)., H.B. and T.I. designed and synthesized Compd-A. T.K. preformed high-throughput chemical library screening with ATPase assay, and kinesin enzymatic assay of Cmpd-A. M.M, H.K. and K.I. evaluated Cmpd-A on pharmacokinetics, toxicity, and physiochemistry, respectively. All authors read and contributed to editing of the manuscript.

\section{Additional information}

Accession codes: The microarray data have been deposited in the Gene Expression Omnibus under accession code GSE67905
Supplementary Information accompanies this paper at http://www.nature.com/ naturecommunications

Competing financial interests: The authors declare no competing financial interests.

Reprints and permission information is available online at http://npg.nature.com/ reprintsandpermissions/

How to cite this article: Ohashi, A. et al. Aneuploidy generates proteotoxic stress and DNA damage concurrently with p53-mediated post-mitotic apoptosis in SAC-impaired cells. Nat. Commun. 6:7668 doi: 10.1038/ncomms8668 (2015).

(c) (i) This work is licensed under a Creative Commons Attribution 4.0 International License. The images or other third party material in this article are included in the article's Creative Commons license, unless indicated otherwise in the credit line; if the material is not included under the Creative Commons license, users will need to obtain permission from the license holder to reproduce the material. To view a copy of this license, visit http://creativecommons.org/licenses/by/4.0/ 